\title{
Sensitivity gradients of surface geometry modifications based on stability analysis of compressible flows
}

\author{
Alejandro Martinez-Cava $\odot,{ }^{1, *}$ Miguel Chávez-Modena $\odot,{ }^{1}$ Eusebio Valero, ${ }^{1,2}$ \\ Javier de Vicente, ${ }^{1,2}$ and Esteban Ferrer $\oplus^{1,2}$ \\ ${ }^{1}$ ETSIAE-UPM, Universidad Politécnica de Madrid, Plaza Cardenal Cisneros 3, E-28040 Madrid, Spain \\ ${ }^{2}$ CCS-UPM - Centre for Computational Simulation - Universidad Politécnica de Madrid, \\ Boadilla del Monte, E-28660 Madrid, Spain
}

(Q) (Received 24 October 2019; accepted 28 May 2020; published 18 June 2020)

\begin{abstract}
We derive a discrete framework for the calculation of eigenvalue sensitivity to geometric deformations. We apply the technique to the steady compressible Navier-Stokes and Reynolds-averaged Navier-Stokes (RANS) flows. The analysis enables one to control (reduce or increase) the amplification rate or frequency associated to the least stable global mode, which is identified using stability analysis. A methodology using a discrete framework is proposed, allowing one to recover the gradients in compressible and turbulent flows. The potential of the resulting shape gradients is evaluated on the well-known circular cylinder flow problem and on a RANS turbulent flow scenario to control the buffet onset on a NACA0012 airfoil. The predicted deformations show excellent performance on the stabilization or excitation, and frequency control, of the global modes and for the two cases tested.
\end{abstract}

DOI: 10.1103/PhysRevFluids.5.063902

\section{INTRODUCTION}

In the search for improved aerodynamic configurations, new tools that couple flow control, stability analysis, and optimization loops are emerging [1-3]. Recent studies [4-6] have developed flow control methodologies using global stability analysis as a source for gradient calculations. Stability analysis studies how small flow perturbations may evolve (naturally or by external agents) and become dominant as time evolves. From a mathematical point of view, stability analyses may demand the resolution of an eigenvalue problem, whose eigenvalues determine the temporal evolution of the spatial perturbations, the associated eigenvectors (or modes). The combination of the obtained eigenpairs with those of the related adjoint eigenvalue problem provides relevant information regarding the response of the system to external modifications or variations of flow parameters or initial conditions [7]. Knowing the influence to variations of additional parameters, such as the turbulent viscosity, Mach or Reynolds number, or the angle of incidence of the incoming flow, is an asset to understanding the underlying physics and design control mechanisms. In this regard, much effort is being addressed to obtain the regions of the flow where the global modes are most sensitive, namely, where small modifications of the flow field could provide the maximum variations on the mode behavior. Of particular importance is the sensitivity to surface modifications, which may be efficiently considered on the improvement of the performance of aerodynamic bodies.

Along these lines, Gianetti and Luchini [8] revisited the concept of the "wavemaker" region in the benchmark problem of the flow around a circular cylinder using global stability analysis. The authors identified the structural sensitivity regions, which is the first step to predict where passive

\footnotetext{
*Corresponding author: alejandro.martinezcava@upm.es
} 
control could be more effective on the delay of the onset of the vortex shedding on the cylinder wake. This phenomenon is widely known to be dominated by a global (absolute) instability. Gianetti and Luchini analytically obtained the optimal areas for the positioning of a small control device, matching the experimental results of Strykowsky and Sreenisavan [9]. Later, Marquet et al. [10] revisited the same flow problem and developed the sensitivity to base flow modifications and steady forcing from a continuous approach. This was later extended by Mettot et al. [11] and Browne et al. [12] for the discrete approach. Additional examples can be found in the work of Meliga et al. [13], who used the sensitivity and adjoint analysis to optimize the position of a heated ring behind an axisymmetric body to control its wake. The same authors studied the sensitivities to boundary forcing on the wake of disks and spheres [14]. Ferrer et al. [15] evaluated the influence of a control cylinder on the wake of an actuator disk, representing a simplified wind turbine, showing relevant changes on the behavior of the unstable mode. More recently, on a planar X-junction, Lashgari et al. [16] optimized the distribution of wall suction and blowing for the first flow bifurcation, also giving a physical explanation for the second bifurcation using the results of the sensitivity analysis. As an example of an eigenvalue-based and gradient-free optimization problem, Wang et al. [3] stabilized the global instability, present on channel contraction, using a genetic-algorithm optimization approach combined with structural sensitivity analysis, which enable one to delay the onset of the instability measured in terms of critical Re number, by 750 percent. This list probably omits many relevant works on flow control based on global stability, so the reader is encouraged to follow the reviews of Luchini [17] and Sipp [18] on adjoint analysis and global stability analysis of open flow configurations. For an extended review of modal analysis, the recent work of Taira et al. [6] illustrates different applications and scopes of this type of analysis.

The extension of linear stability analysis towards surface shape optimization of Navier-Stokes flow fields looks, therefore, natural. Heuveline and Strauß [1] pioneered the use of the least stable eigenvalue as the cost function of an optimization process, implementing a sequential quadratic programming (SQP) methodology for the optimization of the flow over a circular cylinder using finite differences in a continuous approach. Nakazawa and Azegami [19] and, later on, Kiriyama et al. [2], following a continuous approach, proposed a shape optimization problem based on the evaluation of a shape derivative function from an incompressible Navier-Stokes laminar flow field. The use of a Lagrange multiplier method, in which the cost function is the real part of the least stable eigenvalue, has allowed one to optimize open and enclosed flow field configurations. On the stabilization of three-dimensional flows, Tammissola [20] and Tammissola et al. [21] used a second-order perturbation analysis on the optimization of spanwise-periodic shaping and actuation of a cylindrical body to stabilize the vortex shedding. Boujo et al. [22,23] developed an adjoint method to extract the second-order sensitivity of the leading eigenvalues, predicting optimal wall actuation and wall deformation on spanwise-periodic flows, and reducing the complexity of a three-dimensional problem to a two-dimensional analysis. More recently, Brewster and Juniper [24] employed a continuous adjoint approach to obtain the shape gradients of the eigenvalues related to the cylinder wake first instability, and analyze the effects of the local deformations on the flow instability.

In this work, we develop a general discrete framework to compute the gradients (or sensitivities) of a selected global mode (both amplitude and frequency) obtained from the stability analysis of the linearized Navier-Stokes equations. The approach consists of the calculation of the gradients using a discrete approach, which enables easy extension of the approach to any set of equations, including to compressible and turbulent flows [modeled through Reynolds-averaged Navier-Stokes (RANS) approaches]. To do so, the mathematical approach to extract the eigenvalue sensitivity gradients has been revisited. A discrete Lagrangian approach is introduced to calculate the sensitivity to base flow modifications and to the application of a steady forcing, which is subsequently used to obtain the gradients related with a change in a set of flow parameters. The analysis is then extended to geometrical variations, implementing the surface mesh deformation as a control parameter on the Lagrangian formulation. By evaluating the residuals and the Jacobian matrix of the flow solution with respect to modifications in the surface mesh coordinates, we are able to obtain the gradients 
related with the surface mesh deformation. Previous work in the literature required one evaluation per geometrical design variable. A major improvement of our approach is that the sensitivity of the eigenvalue with respect to the movement of all the surface mesh nodes is obtained in one single evaluation, but retaining individual information for each mesh node. The sensitivity gradients can be used to guide local deformations to control the growth rate or associated frequency of the eigenvalue, leading to a control of the physical phenomena at play.

This methodology is validated for the laminar flow over a circular cylinder, where unsteady vortex shedding appears at Reynolds numbers (based on the cylinder diameter and the free stream velocity) beyond $\operatorname{Re}=46$ [25]. The global mode can be either stabilized, delaying the onset of the vortex shedding, or controlled to modulate the associated frequency employing the proposed methodology. In addition, the extension of the proposed eigenvalue sensitivity to compressible and turbulent flows is evaluated for a transonic flow over a NACA0012 airfoil. At these flow conditions, the problem is dominated by the onset of buffet, resulting on oscillating aerodynamic loads that can be detrimental to the structural safety of the wing [26]. The application of the sensitivity gradients enables the local deformation of the suction side of the airfoil to suppress the absolute instability responsible for the buffet.

The rest of the paper is divided into four sections. Section II reviews the mathematical modeling comprising the stability analysis and the sensitivities calculation, and Sec. III extends the implementation and verification of the algorithm with an analytic test case. Section IV presents the validation of the methodology on the control of the cylinder wake, with Sec. V presenting the results of the analysis and control of the buffet instability on a NACA0012 airfoil. The paper ends with conclusions in Sec. VI.

\section{MATHEMATICAL MODEL}

\section{A. Stability analysis}

We present a general notation for the partial differential equations (PDE) system (including boundary conditions) that encompasses the compressible Navier-Stokes equations and RANS equations for turbulent regimes (e.g., Spalart-Allmaras model [27]). Both can be written in conservative form as

$$
\mathbf{M} \frac{\partial \mathbf{q}}{\partial t}=\mathbf{R}(\mathbf{q})
$$

where $\mathbf{q}$ defines the conservative variables, $\mathbf{M}$ is the mass matrix of the system, and $\mathbf{R}(\mathbf{q})$ is the discrete spatial nonlinear operator, comprising inviscid and viscous terms. Once discretized, vectors $\mathbf{q}$ and $\mathbf{R}(\mathbf{q})$ have dimensions $N_{v} \times N$, where $N_{v}$ is the number of fluid variables considered and $N$ is the number of nodes of the discretization method. Without loss of generality, solutions of Eq. (1) can be decomposed into a steady component upon which a small perturbation is superimposed,

$$
\mathbf{q}(\mathbf{x}, t)=\overline{\mathbf{q}}(\mathbf{x})+\varepsilon \widetilde{\mathbf{q}}(\mathbf{x}, t),
$$

where $\varepsilon \ll 1$ and $\overline{\mathbf{q}}$ is the so-called base flow, steady point of equilibrium of Eq. (1).

This decomposition is introduced into the system (1) and a Taylor series expansion is performed around the point of equilibrium $\overline{\mathbf{q}}$. Neglecting terms of the order of $\varepsilon^{2}$ and assembling all the unknowns in vector-matrix notation, we obtain

$$
\varepsilon \mathbf{M} \frac{\partial \widetilde{\mathbf{q}}}{\partial t}=\mathbf{R}(\overline{\mathbf{q}}+\varepsilon \widetilde{\mathbf{q}}) \approx \mathbf{R}(\overline{\mathbf{q}})+\varepsilon\left[\frac{\partial \mathbf{R}(\mathbf{q})}{\partial \mathbf{q}}\right]_{\overline{\mathbf{q}}} \widetilde{\mathbf{q}},
$$

where $\mathbf{M}$ is the mass matrix resulting from the spatial discretization, $[\partial \mathbf{R}(\mathbf{q}) / \partial \mathbf{q}]_{\overline{\mathbf{q}}}$ represents the Jacobian matrix of the fluxes evaluated for the base flow, and $\mathbf{R}(\overline{\mathbf{q}})=0$ for steady flows. For finite volume approximations (see Sec. III A), the mass matrix $\mathbf{M}$ is diagonal with leading dimension $N_{v} \times N$ and contains the volumes associated to each cell. 
In the two-dimensional context of the problems addressed in the present work, we consider perturbations under the form of two-dimensional (2D) normal modes,

$$
\widetilde{\mathbf{q}}(\mathbf{x}, t)=\widehat{\mathbf{q}}(x, z) e^{\sigma t},
$$

where $\sigma$ is a complex scalar magnitude (eigenvalue) and $\widehat{\mathbf{q}}$ describes the spatial complex disturbance (eigenmode), a function of the spatial coordinates $x$ and $z$. Linearizing around the point of equilibrium of Eq. (1), we can finally recover the eigenvalue problem,

$$
\mathbf{A}(\overline{\mathbf{q}}) \widehat{\mathbf{q}}=\sigma \widehat{\mathbf{q}},
$$

with $\mathbf{A}(\overline{\mathbf{q}})=\mathbf{M}^{-1}[\partial \mathbf{R}(\mathbf{q}) / \partial \mathbf{q}]_{\overline{\mathbf{q}}}$ being the Jacobian, a square matrix of leading dimension $N_{v} \times N$, and $\sigma=\sigma_{r}+i \sigma_{i}$ representing the complex eigenvalues of the system. The real part of the eigenvalue, $\sigma_{r}$, refers to the amplification rate of the corresponding eigenmode, with the imaginary part $\sigma_{i}$ being the pulsation, related to the associated Strouhal number as $\mathrm{St}=\sigma_{i} / 2 \pi$.

The adjoint eigenvalue problem is defined using the adjoint operator $\mathbf{A}^{+}(\overline{\mathbf{q}})$ as

$$
\mathbf{A}^{+}(\overline{\mathbf{q}}) \widehat{\mathbf{q}}^{+}=\sigma^{+} \widehat{\mathbf{q}}^{+}
$$

with the complex vector $\widehat{\mathbf{q}}^{+}$being the adjoint eigenvectors, and $\sigma^{+}$the adjoint eigenvalue, complex conjugate of the eigenvalue of system (5). Relying on the discrete inner product $\langle\mathbf{u}, \mathbf{v}\rangle=\mathbf{u}^{T} \mathbf{M v}$, the adjoint operator of the Jacobian matrix [7,17] can be obtained as $\mathbf{A}^{+}(\overline{\mathbf{q}})=\mathbf{M}^{-1} \mathbf{A}^{H}(\overline{\mathbf{q}}) \mathbf{M}$, where the superindex $H$ denotes the Hermitian transpose. It is worth mentioning that if the linear operator A was defined as $[\partial \mathbf{R}(\mathbf{q}) / \partial \mathbf{q}]_{\overline{\mathbf{q}}}$ (not scaled by matrix $\mathbf{M}$ ), Eq. (5) would result in a generalized eigenvalue problem, with the adjoint operator formulated as $\mathbf{A}^{H}$. The adjoint eigenmodes would be recovered calculating the left eigenvectors [28]. In the present manuscript, the formulation presented in Eqs. (5) and (6) is retained.

\section{B. Sensitivity analysis to geometrical modifications}

Stability analysis studies the growth or decay of perturbations superimposed onto a usually steady solution of the Navier-Stokes equations. The analysis can identify particular flow features and their evolution under slight modifications of the flow conditions, either by introducing a perturbation or by a modification of some physical or geometrical parameter. The growth of these features gives rise to a different flow configuration. The stability of the system can be analyzed solving the derived eigenvalue problem [Eq. (5)], obtaining the relevant (physical) eigenmodes that would drive the system towards an unstable configuration.

To complement stability analyses, sensitivity analyses provide information on the response of the system to variations of parameters, either physical, geometrical, or to external perturbations, normally evaluated through the variation of a target functional. In our case, we are interested in the sensitivity of the eigenvalue and to assess how it will respond to the modification of chosen parameters or conditions of the flow configuration. Our analysis focuses on how the eigenvalues of the system, $\sigma \equiv \sigma(\overline{\mathbf{q}}(\mathbf{p})$, $\mathbf{p})$, will respond to variations of $\mathbf{p}$, the parameter (or parameters) of interest. We assume that our system of PDEs has a unique solution for each value of $\mathbf{p}$, and every variable is at least $C^{2}$.

The method of Lagrange multipliers is used to calculate $\nabla_{\mathbf{p}} \sigma$ for a range of parameters, considering as well the different physical constraints of the problem. In the most general framework, the Lagrangian functional is defined as

$$
\mathcal{L}\left(\overline{\mathbf{q}}, \sigma, \widehat{\mathbf{q}}, \mathbf{p}, \lambda_{1}, \lambda_{2}\right)=\sigma+\left\langle\lambda_{1}, \mathbf{A}(\overline{\mathbf{q}}, \mathbf{p}) \widehat{\mathbf{q}}-\sigma \widehat{\mathbf{q}}\right\rangle+\left\langle\lambda_{2}, \mathbf{M}^{-1} \mathbf{R}(\overline{\mathbf{q}}, \mathbf{p})\right\rangle,
$$

where, as it is shown in the Appendix, $\lambda_{1}$ and $\lambda_{2}$ are the Lagrange multipliers that represent the adjoint eigenmode $\widehat{\mathbf{q}}^{+}$and adjoint base flow $\overline{\mathbf{q}}^{+}$, respectively. Vector $\mathbf{p}$, of dimension $N_{p}$, contains the parameters of interest to the sensitivity analysis. The gradient of the Lagrangian can be expressed 
as

$$
\nabla \mathcal{L}=\left\langle\frac{\partial \mathcal{L}}{\partial \overline{\mathbf{q}}}, \delta \overline{\mathbf{q}}\right\rangle+\left\langle\frac{\partial \mathcal{L}}{\partial \sigma}, \delta \sigma\right\rangle+\left\langle\frac{\partial \mathcal{L}}{\partial \widehat{\mathbf{q}}}, \delta \widehat{\mathbf{q}}\right\rangle+\left\langle\frac{\partial \mathcal{L}}{\partial p}, \delta \mathbf{p}\right\rangle+\left\langle\frac{\partial \mathcal{L}}{\partial \lambda_{1}}, \delta \lambda_{1}\right\rangle+\left\langle\frac{\partial \mathcal{L}}{\partial \lambda_{1}}, \delta \lambda_{2}\right\rangle
$$

Differentiation with respect to the Lagrange multipliers recovers the two constraints of the problem (base flow computation and eigenvalue problem), and the differentiation with respect to the eigenmode leads to the adjoint eigenvalue problem, respectively. In addition, the derivation with respect to the eigenvalue leads to the dimensionalization condition of the adjoint eigenmode, $\left\langle\widehat{\mathbf{q}}^{+}, \widehat{\mathbf{q}}\right\rangle=1$.

When canceling the derivative with respect to the base flow, we recover the linear system,

$$
\mathbf{A}(\overline{\mathbf{q}}, \mathbf{p})^{+} \overline{\mathbf{q}}^{+}=-\nabla_{\overline{\mathbf{q}}} \sigma
$$

with

$$
\begin{gathered}
\nabla_{\overline{\mathbf{q}}} \sigma=\mathbf{B}^{+}(\widehat{\mathbf{q}}, \mathbf{p}) \widehat{\mathbf{q}}^{+}, \\
\nabla_{\mathbf{q}_{\mathbf{f}}} \sigma=\overline{\mathbf{q}}^{+},
\end{gathered}
$$

where $\nabla_{\overline{\mathbf{q}}} \sigma$ is the sensitivity of the eigenvalue to base flow modifications, and $\nabla_{\mathbf{q}_{\mathbf{f}}} \sigma$ is the sensitivity to steady forcing, respectively, with both previously derived by Marquet et al. [10] and later extended by Browne et al. [12] and Mettot et al. [11] in a discrete framework. The operator $\mathbf{B}(\widehat{\mathbf{q}}, \mathbf{p})=\partial(\mathbf{A}(\overline{\mathbf{q}}, \mathbf{p}) \widehat{\mathbf{q}}) / \partial \overline{\mathbf{q}}$ is the so-called Hessian complex matrix, and $\overline{\mathbf{q}}^{+}$is defined as the adjoint operator of the base flow, obtained from the solution of the linear system (9). For completeness, we include in the Appendix the detailed development of the sensitivity gradients in a discrete framework, which can also be found in Browne et al. [12]. It is worth mentioning that for the sensitivity analysis of steady and mean flows, the operator $\mathbf{B}$ contains no explicit dependence on the base flow $\overline{\mathbf{q}}$. However, in more general cases, the sensitivity may depend explicitly on the base flow, e.g., when deriving sensitivities associated to secondary instabilities [29].

Finally, the derivative of the Lagrangian with respect to a change in the parameters is obtained via the expression

$$
\begin{aligned}
\left\langle\frac{\partial \mathcal{L}}{\partial \mathbf{p}}, \delta \mathbf{p}\right\rangle & =\left\langle\widehat{\mathbf{q}}^{+}, \frac{\partial \mathbf{A}(\overline{\mathbf{q}}, \mathbf{p}) \widehat{\mathbf{q}}}{\partial \mathbf{p}} \delta \mathbf{p}\right\rangle+\left\langle\overline{\mathbf{q}}^{+}, \mathbf{M}^{-1} \frac{\partial \mathbf{R}(\overline{\mathbf{q}}, \mathbf{p})}{\partial \mathbf{p}} \delta \mathbf{p}\right\rangle \\
& =\left\langle\widehat{\mathbf{q}}^{+}, \mathbf{B}_{\mathbf{p}}(\widehat{\mathbf{q}}, \mathbf{p}) \delta \mathbf{p}\right\rangle+\left\langle\overline{\mathbf{q}}^{+}, \mathbf{A}_{\mathbf{p}}(\overline{\mathbf{q}}, \mathbf{p}) \delta \mathbf{p}\right\rangle \\
& =\left\langle\mathbf{B}_{\mathbf{p}}{ }^{+}(\widehat{\mathbf{q}}, \mathbf{p}) \widehat{\mathbf{q}}^{+}+\mathbf{A}_{\mathbf{p}}{ }^{+}(\overline{\mathbf{q}}, \mathbf{p}) \overline{\mathbf{q}}^{+}, \delta \mathbf{p}\right\rangle
\end{aligned}
$$

As $\mathbf{p}$ is a vector of dimension $N_{p}, \mathbf{B}_{\mathbf{p}}(\widehat{\mathbf{q}}, \mathbf{p})=\partial(\mathbf{A}(\overline{\mathbf{q}}, \mathbf{p}) \widehat{\mathbf{q}}) / \partial \mathbf{p}$ and $\mathbf{A}_{\mathbf{p}}(\overline{\mathbf{q}}, \mathbf{p})=\mathbf{M}^{-1} \partial \mathbf{R}(\overline{\mathbf{q}}, \mathbf{p}) / \partial \mathbf{p}$ will be complex and real matrices, respectively, of dimension $\left(N_{v} \times N\right) \times N_{p}$, once the problem is discretized. With the term $\overline{\mathbf{q}}^{+}$present in the right-hand side of (12), the calculation of the sensitivity to base flow modifications and the forcing term are a necessary step to obtain the sensitivity to a change in the parameters.

Since the last two constraints in the right-hand side terms of Eq. (7) are identically zero, the gradient of $\sigma$ with respect to variations of vector $\mathbf{p}$ can be obtained as

$$
\Delta \sigma=\left\langle\nabla_{\mathbf{p}} \sigma, \delta \mathbf{p}\right\rangle=\left\langle\frac{\partial \mathcal{L}}{\partial \mathbf{p}}, \delta \mathbf{p}\right\rangle
$$

Expanding the inner products of Eqs. (12) and (13), and making use of the adjoint operator, we finally arrive at

$$
\nabla_{\mathbf{p}} \sigma=\mathbf{M}^{-1} \mathbf{B}_{\mathbf{p}}{ }^{H}(\widehat{\mathbf{q}}, \mathbf{p}) \mathbf{M} \widehat{\mathbf{q}}^{+}+\mathbf{M}^{-1} \mathbf{A}_{\mathbf{p}}{ }^{H}(\overline{\mathbf{q}}, \mathbf{p}) \mathbf{M} \overline{\mathbf{q}}^{+}
$$




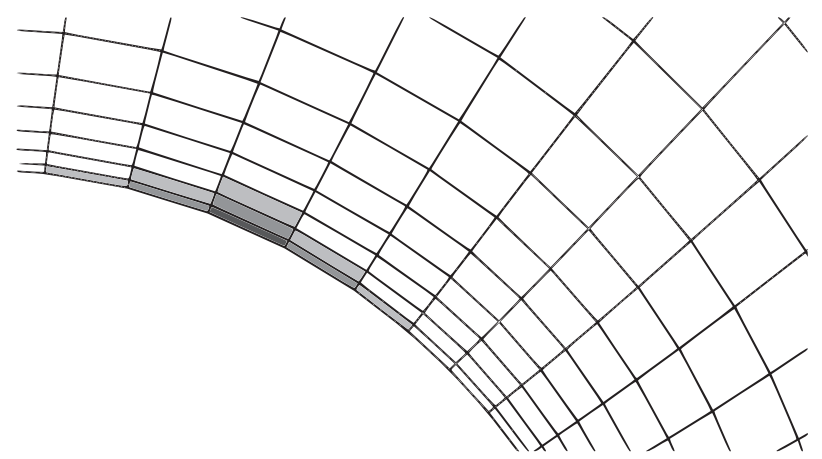

FIG. 1. Compact second-order stencil used on the calculation of the Jacobian and the residuals. The darkgray cell indicates the wall cell that is deformed, gray cells correspond to the immediate neighbors, and lightgray cells are the immediate next neighbors.

Alternatively, using the chain rule, the response of the eigenvalue with respect to changes of the parameters of interest could be easily defined as

$$
\frac{d \sigma}{d \mathbf{p}}=\frac{\partial \sigma}{\partial \overline{\mathbf{q}}} \frac{\partial \overline{\mathbf{q}}}{\partial \mathbf{p}}+\frac{\partial \sigma}{\partial \mathbf{p}} .
$$

This equation is equivalent to (14), where the first term represents the variation induced by $\mathbf{p}$ on the base flow, and the second one the explicit dependence of the eigenvalue $\sigma$ with $\mathbf{p}$. Obviously, for a small number of parameters, it is possible and cheaper to apply the chain rule, given by Eq. (15), to compute the sensitivity. However, for a large number of parameters (e.g., mesh nodes on a surface geometry), it is much more efficient to use the adjoint base approach given by Eq. (14).

In particular, we are interested in the effect that a displacement of a set of surface mesh nodes would have on a particular eigenvalue. Therefore, we propose substituting $\delta \mathbf{p}$ by the sparse vector $\delta \mathbf{X}$, which contains the perturbation produced by the infinitesimal displacement of a set of surface mesh nodes. Vector $\delta \mathbf{X}$ has leading dimension $N_{p}=N_{v} \times N$, with the nonzero elements being defined by the contributions of the displaced mesh nodes and those affected by the second-order stencil of the numerical method (Fig. 1). $\nabla_{\mathbf{p}} \sigma$, now renamed as $\nabla_{\mathbf{X}} \sigma$, is a vector with the same dimension, $N_{p}$, containing information relative to the sensitivity of the eigenvalue to the displacement of a set of mesh nodes. Equation (14) is reformulated for clarity as

$$
\nabla_{\mathbf{X}} \sigma=\mathbf{M}^{-1} \mathbf{B}_{\mathbf{p}}(\widehat{\mathbf{q}}, \mathbf{X})^{H} \mathbf{M} \widehat{\mathbf{q}}^{+}+\mathbf{M}^{-1} \mathbf{A}_{\mathbf{p}}(\overline{\mathbf{q}}, \mathbf{X})^{H} \mathbf{M} \overline{\mathbf{q}}^{+}
$$

This expression can be compared with the analysis performed by Brewster and Juniper [24], who derived a similar expression in the continuous setting for incompressible flows. They recover two terms in their sensitivity gradients: (1) a feedback contribution eigenvalue response caused by changes in the unsteady feedback mechanism of the global mode, which can be related with the second term of Eq. (16), and (2) a base flow contribution caused by the changes in the base flow originated by the surface deformation, which is the first term of Eq. (16).

\section{IMPLEMENTATION AND VALIDATION}

\section{A. Numerical implementation and methodology}

The DLR TAU code [30] (referred to as TAU from now on) is used for the computations. TAU is a computational fluid dynamics (CFD) solver for external aerodynamic calculations, based on a second-order finite volume method for unstructured grids and a compressible flow approximation. In this work, TAU is used to calculate the laminar and/or turbulent, steady base flow solutions. Given a particular flow solution, the code permits the direct extraction of an analytic version of 
the Jacobian matrix $\mathbf{A}(\overline{\mathbf{q}}, \mathbf{X})$ following a first-discretize-then-linearize strategy, together with the residual vector $\mathbf{R}(\overline{\mathbf{q}}, \mathbf{X})$. The Jacobian matrix is hand derived and hard coded into the TAU, including the linearization of one- and two-equation turbulence models. Within the discrete framework, boundary conditions are embedded in the linear operator and do not need to be evaluated separately. In TAU, no approximation of the boundary conditions is required, as all are treated numerically with a stencil of a single (boundary) point, and therefore appear only on the diagonal of the Jacobian. This has been documented in Dwight [31], who states that the Jacobian and its discrete adjoint have identical eigenspectra, without the presence of any spurious eigenvalue in the adjoint, as reported in other works; e.g., Juniper [28]. The eigenvalues and eigenvectors of the eigenvalue problem, given by Eq. (5), are obtained applying an Arnoldi method, combined with a shift-and-invert spectral transformation. To solve the linear systems associated with the iterative generation of the Krylov subspace, a full lower-upper (LU) decomposition of the sparse matrix $\mathbf{A}(\overline{\mathbf{q}}, \mathbf{X})$ is calculated in parallel and distributed memory, using the MUMPS package [32]. This methodology has been validated in different scenarios to recover the physical eigenvalues; see, for instance, [3,11,3335]. In this work, the calculation of eigenvalues involves a Krylov-Schur technique, implemented in the public numerical library SLEPc [36]. The matrix-based approach has been chosen to avoid missing important eigenvalue branches, as the most important eigenvalues are the first to converge in a discrete framework [37]. This approach has, however, the drawback of the memory limitations related to the matrix storage, as the scaling of the full LU decomposition behaves as $\left(N_{v} \times N\right)^{3}$ [38]. However, domain reduction strategies [39] and efficient matrix distribution [40] reduce memory demands and allows one to perform stability analyses of three-dimensional configurations [3].

Since TAU only provides the Jacobian matrix, we consider the following procedure to compute the second derivatives for the Hessian, given by Eq. (10). From its definition, it follows that $\mathbf{B}(\widehat{\mathbf{q}}, \mathbf{X})$ is a linear function of $\widehat{\mathbf{q}}=\widehat{\mathbf{q}}_{R}+i \widehat{\mathbf{q}}_{I}$ and a nonlinear function of $\overline{\mathbf{q}}$ through the Jacobian of $\mathbf{R}(\overline{\mathbf{q}}, \mathbf{X})$. Thus, it can be decomposed into its real and imaginary parts,

$$
\mathbf{B}(\widehat{\mathbf{q}}, \mathbf{X})=\mathbf{B}(\widehat{\mathbf{q}}, \mathbf{X})_{R}+i \mathbf{B}(\widehat{\mathbf{q}}, \mathbf{X})_{I}=\frac{\partial \mathbf{A}(\overline{\mathbf{q}}, \mathbf{X}) \widehat{\mathbf{q}}_{R}}{\partial \overline{\mathbf{q}}}+i \frac{\partial \mathbf{A}(\overline{\mathbf{q}}, \mathbf{X}) \widehat{\mathbf{q}}_{I}}{\partial \overline{\mathbf{q}}} .
$$

Applying the definition of the directional derivative, and neglecting terms of the order of $O\left(\epsilon_{1}^{2}\right)$, the calculation of the sensitivity matrix is recovered as

$$
\mathbf{B}(\widehat{\mathbf{q}}, \mathbf{X})=\frac{\partial \mathbf{A}(\overline{\mathbf{q}}, \mathbf{X}) \widehat{\mathbf{q}}}{\partial \overline{\mathbf{q}}}=\frac{1}{\epsilon_{1}}\left[\mathbf{A}\left(\overline{\mathbf{q}}+\epsilon_{1} \widehat{\mathbf{q}}, \mathbf{X}\right)-\mathbf{A}(\overline{\mathbf{q}}, \mathbf{X})\right] .
$$

For a detailed analysis on the influence of $\epsilon_{1}$, the reader is referred to the work of Browne et al. [12]. Having computed the Jacobian and Hessian matrices, it is possible to obtain the sensitivity to base flow and external forcing following Eqs. (10) and (11), as described in the Appendix.

In the cases where only one mesh node is considered for the sensitivity analysis, Eq. (16) will give the sensitivity of the eigenvalue to a normal displacement. A matrix could be obtained by repeating this process for every single mesh node, but note that this process would be computationally expensive. To evaluate the sensitivity to mesh deformations, we aim to eliminate the need of evaluating the sensitivity of the eigenvalue for every single mesh node, and hence we propose to collect all the information to evaluate all deformations in only one iteration. To this aim, the surface nodes are displaced a distance $\epsilon_{2}$ in their corresponding surface normal direction (all at once), which leads to $\mathbf{A}_{\mathbf{p}}(\overline{\mathbf{q}}, \mathbf{X})$ and $\mathbf{B}_{\mathbf{p}}(\widehat{\mathbf{q}}, \mathbf{X})$ to be vectors of dimension $N_{v} \times N$. The extension of this modification will only generate an effect on the neighbor nodes affected by the second-order stencil of the finite volume solver (Fig. 1), and the value of $\epsilon_{2}$ should be small enough to not induce a collapse of the first layer of the boundary layer volume mesh, whose cells may suffer from having a high aspect ratio. A detailed sensitivity study to the value of $\epsilon_{2}$ is presented in Sec. III B. The new residual vector and Jacobian matrix can be calculated using the new mesh (and keeping the old flow solution), such as $\mathbf{R}\left(\overline{\mathbf{q}}, \mathbf{X}+\epsilon_{2} \mathbf{d}\right)$ and $\mathbf{A}\left(\overline{\mathbf{q}}, \mathbf{X}+\epsilon_{2} \mathbf{d}\right)$, with $\mathbf{d}$ a vector of dimension $N_{v} \times N$ with the rows corresponding to the surface nodes set to 1 , and 0 in the other entries. 


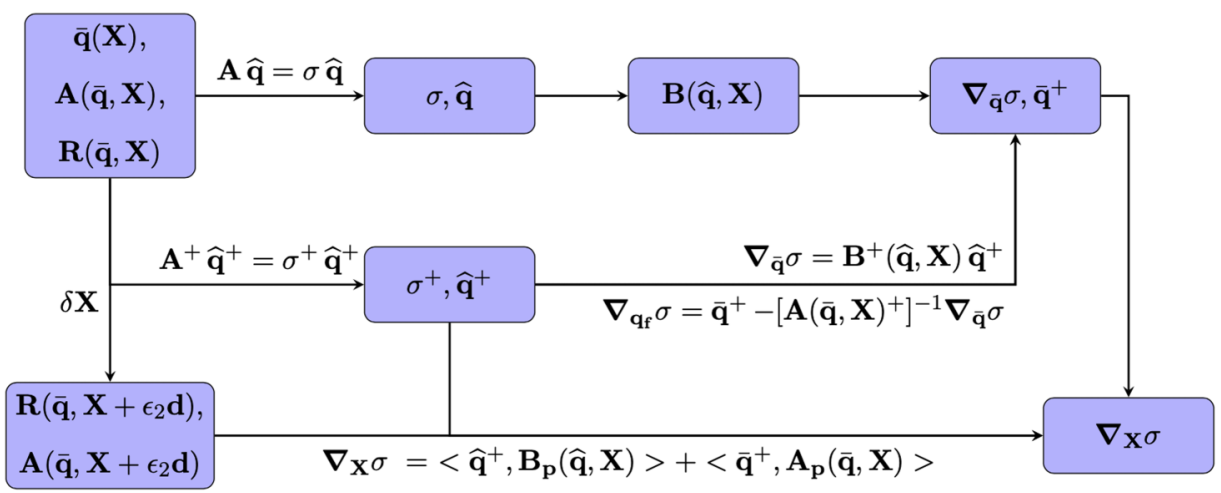

FIG. 2. Mathematical procedure for the calculation of the sensitivity to mesh node displacements.

With $\mathbf{M}(\mathbf{X})$ and $\mathbf{M}\left(\mathbf{X}+\epsilon_{\mathbf{2}} \mathbf{d}\right)$ the mass matrices for the original and the deformed meshes, the calculation of vector $\mathbf{A}_{\mathbf{p}}(\overline{\mathbf{q}}, \mathbf{X})$ is then performed as

$$
\mathbf{A}_{\mathbf{p}}(\overline{\mathbf{q}}, \mathbf{X})=\frac{\mathbf{M}\left(\mathbf{X}+\epsilon_{2} \mathbf{d}\right)^{-1} \mathbf{R}\left(\overline{\mathbf{q}}, \mathbf{X}+\epsilon_{2} \mathbf{d}\right)-\mathbf{M}(\mathbf{X})^{-1} \mathbf{R}(\overline{\mathbf{q}}, \mathbf{X})}{\epsilon_{2}},
$$

while for vector $\mathbf{B}_{\mathbf{p}}(\widehat{\mathbf{q}}, \mathbf{X})$,

$$
\mathbf{B}_{\mathbf{p}}(\widehat{\mathbf{q}}, \mathbf{X})=\frac{\left[\mathbf{A}\left(\overline{\mathbf{q}}, \mathbf{X}+\epsilon_{2} \mathbf{d}\right)-\mathbf{A}(\overline{\mathbf{q}}, \mathbf{X})\right] \widehat{\mathbf{q}}}{\epsilon_{2}} .
$$

The resulting vectors in Eqs. (19) and (20) comprise nonzero elements only in those positions related with the surface mesh nodes of interest and their stencil. The discrete gradients $\nabla_{\mathbf{X}} \sigma$ are recovered by the discrete inner product, $\langle\bullet, \bullet\rangle$, of vectors $\mathbf{A}_{\mathbf{p}}(\overline{\mathbf{q}}, \mathbf{X})$ and $\mathbf{B}_{\mathbf{p}}(\widehat{\mathbf{q}}, \mathbf{X})$ with the adjoint base flow and adjoint global mode, respectively,

$$
\nabla_{\mathbf{X}} \sigma=\left\langle\widehat{\mathbf{q}}^{+}, \mathbf{B}_{\mathbf{p}}(\widehat{\mathbf{q}}, \mathbf{X})\right\rangle+\left\langle\overline{\mathbf{q}}^{+}, \mathbf{A}_{\mathbf{p}}(\overline{\mathbf{q}}, \mathbf{X})\right\rangle .
$$

The obtained final vector $\nabla_{\mathbf{X}} \sigma$ contains information relative to the sensitivity of the eigenvalue with respect to the normal displacement of each one of the displaced surface mesh nodes, gathered in just one computational evaluation.

To sum up, the procedure to obtain the sensitivity gradients to geometrical changes is depicted in Fig. 2, which can be itemized in the following steps:

(1) Calculate the base flow $\overline{\mathbf{q}}$ using the Navier-Stokes solver.

(2) Compute the direct global modes $\widehat{\mathbf{q}}$ based on the discrete Jacobian, $\mathbf{A}(\overline{\mathbf{q}}, \mathbf{X})$.

(3) Compute the adjoint global modes $\widehat{\mathbf{q}}^{+}$based on the discrete adjoint operator of the Jacobian, $\mathbf{A}^{+}(\overline{\mathbf{q}}, \mathbf{X})=\mathbf{M}^{-1} \mathbf{A}(\overline{\mathbf{q}}, \mathbf{X}) \mathbf{M}$.

(4) Calculate the sensitivity to base flow modifications, $\nabla_{\overline{\mathbf{q}}} \sigma=\mathbf{B}^{+}(\widehat{\mathbf{q}}, \mathbf{X}) \widehat{\mathbf{q}}^{+}$.

(5) Solve the linear system $\mathbf{A}(\overline{\mathbf{q}}, \mathbf{X})^{+} \overline{\mathbf{q}}^{+}=-\nabla_{\overline{\mathbf{q}}} \sigma$ to obtain the sensitivity to steady forcing, $\nabla_{\mathbf{q}_{\mathrm{f}}} \sigma=\overline{\mathbf{q}}^{+}$.

(6) Extract the residuals vector of the flow solution, $\mathbf{R}(\overline{\mathbf{q}}, \mathbf{X})$.

(7) Displace the surface mesh nodes in normal direction by a small distance $\epsilon_{2} \ll 1$.

(8) Compute the new residuals vector and Jacobian matrix, $\mathbf{R}\left(\overline{\mathbf{q}}, \mathbf{X}+\epsilon_{2} \mathbf{d}\right)$ and $\mathbf{A}\left(\overline{\mathbf{q}}, \mathbf{X}+\epsilon_{2} \mathbf{d}\right)$, followed by the matrix $\mathbf{B}_{\mathbf{p}}(\widehat{\mathbf{q}}, \mathbf{X})$ and the vector $\mathbf{A}_{\mathbf{p}}(\overline{\mathbf{q}}, \mathbf{X})$, using Eqs. (19) and (20).

(9) Calculate the sensitivity to geometrical changes, $\nabla_{\mathbf{X}} \sigma$, using Eq. (21).

\section{B. Analytic test case}

To check the validity of our expressions, we consider a simplified analytic test case with two flow variables, $u$ and $v$, and one parameter $p$. Therefore, $\mathbf{q}=(u, v, p)$ is the state vector, and the 
corresponding flux vector defined by the nonlinear system,

$$
\mathbf{R}(\mathbf{q}, p)=\left[\begin{array}{c}
e^{p u v}-1 \\
u p^{2}+v+1
\end{array}\right]
$$

and the system mass matrix arbitrarily defined as

$$
\mathbf{M}=\left[\begin{array}{cc}
0.25 & 0.0 \\
0.0 & 0.5
\end{array}\right]
$$

System (22) has a point of equilibrium (base flow) $\overline{\mathbf{q}}=(\bar{u}, \bar{v}, p)=\left(-1 / p^{2}, 0, p\right)$, for which $\mathbf{R}(\overline{\mathbf{q}}, p)=\mathbf{0}$, upon which the stability analysis is computed. The analytic Jacobian matrix with respect to that base flow $(\bar{u}, \bar{v})$ can be easily calculated,

$$
\mathbf{A}(\overline{\mathbf{q}}, p)=\mathbf{M}^{-1}[\partial \mathbf{R}(\mathbf{q}, p) / \partial \mathbf{q}]_{\mathbf{q}=\overline{\mathbf{q}}}=\mathbf{M}^{-1}\left[\begin{array}{cc}
p v e^{p u v} & p u e^{p u v} \\
p^{2} & 1
\end{array}\right]_{\mathbf{q}=\overline{\mathbf{q}}}=\left[\begin{array}{cc}
0 & -4 / p \\
2 p^{2} & 1
\end{array}\right] .
$$

When we consider the eigenvalue problem $\mathbf{A}(\overline{\mathbf{q}}, p) \widehat{\mathbf{q}}=\sigma \widehat{\mathbf{q}}$, the eigenvalues are $\sigma_{1,2}=(1 \pm$ $\sqrt{1-8 p}$ ). After some calculations, the direct and adjoint global modes, $\widehat{\mathbf{q}}^{+}, \overline{\mathbf{q}}^{+}$, and matrices $\mathbf{B}_{\mathbf{p}}(\widehat{\mathbf{q}}, p), \mathbf{A}_{\mathbf{p}}(\overline{\mathbf{q}}, p)$ are obtained:

$$
\begin{aligned}
& \mathbf{B}(\widehat{\mathbf{q}}, p)=\left[\begin{array}{cc}
4 e^{p u v} p^{2}\left[\widehat{\mathbf{q}}_{1} v^{2}+\widehat{\mathbf{q}}_{2}(u v+1 / p)\right] & 4 e^{p u v} p^{2}\left[\widehat{\mathbf{q}}_{2} u^{2}+\widehat{\mathbf{q}}_{1}(u v+1 / p)\right] \\
0 & 0
\end{array}\right]_{\mathbf{q}=\overline{\mathbf{q}}}, \\
& \mathbf{B}_{\mathbf{p}}(\widehat{\mathbf{q}}, p)=\left[\begin{array}{c}
4 e^{p u v}\left(v \widehat{\mathbf{q}}_{1}+u \widehat{\mathbf{q}}_{2}\right)(p u v+1) \\
4 p \widehat{\mathbf{q}}_{1}
\end{array}\right]_{\mathbf{q}=\overline{\mathbf{q}}}, \quad \mathbf{A}_{\mathbf{p}}(\overline{\mathbf{q}}, p)=\mathbf{M}^{-1}\left[\begin{array}{c}
u v e^{p u v} \\
2 p u
\end{array}\right]_{\mathbf{q}=\overline{\mathbf{q}}},
\end{aligned}
$$

where $\widehat{\mathbf{q}}=\left(\widehat{\mathbf{q}}_{1}, \widehat{\mathbf{q}}_{2}\right)$ is the associated eigenvector. Finally, to compute the sensitivity of the eigenvalue $\sigma_{1}$ to changes in $p$ (in this case, it can either be computed analytically using the chain rule or the Lagrangian approach), we use

$$
\nabla_{p} \sigma_{\text {Analytic }}=\frac{\mathrm{d} \sigma_{1}}{\mathrm{~d} p}=\frac{\partial \sigma_{1}}{\partial \overline{\mathbf{q}}} \frac{\partial \overline{\mathbf{q}}}{\partial p}+\frac{\partial \sigma_{1}}{\partial p}=-\frac{4}{\sqrt{1-8 p}} .
$$

At this point, we select a range for $p>1 / 8$ such that the resulting eigenvalues are always complex, which is often what happens in more realistic conditions (see Sec. IV). We evaluate the sensitivity of the numerical methodology described in Sec. III A to the values chosen for the $\epsilon_{2}$ parameter. To do so, the relative error is calculated as

$$
E=\left|\frac{\nabla_{p} \sigma_{\text {Analytic }}-\nabla_{p} \sigma_{\text {Numerical }}}{\nabla_{p} \sigma_{\text {Analytic }}}\right|
$$

where $\nabla_{p} \sigma_{\text {Numerical }}$ has been calculated with the numerical version of matrices $\mathbf{B}_{\mathbf{p}}(\widehat{\mathbf{q}}, p), \mathbf{A}_{\mathbf{p}}(\overline{\mathbf{q}}, p)$ using Eqs. (19) and (20). The results are shown in Fig. 3, where it can be observed that optimal accuracy is reached for values of $\epsilon_{2}$ of the order of $O\left(10^{-7}\right)$, and that slight variations of $\epsilon_{2}$ will still keep the error low.

\section{NUMERICAL VALIDATION. THE CYLINDER WAKE ABSOLUTE INSTABILITY}

\section{A. Case description}

The first instability of the cylinder wake has been widely investigated using global stability analysis $[8,10,25,41,42]$. For a critical Reynolds number $(\operatorname{Re} \approx 46)$, an antisymmetrical global mode becomes unstable, increasing the intensity of the perturbations inside the recirculation region of the wake until its symmetry breaks, developing the well-known von Karman vortex street. We revisit this case as a two-dimensional laminar flow numerical experiment, aiming to evaluate the behavior of the cylinder wake global mode, when the body surface is modified, using the proposed global mode sensitivity gradients approach. 


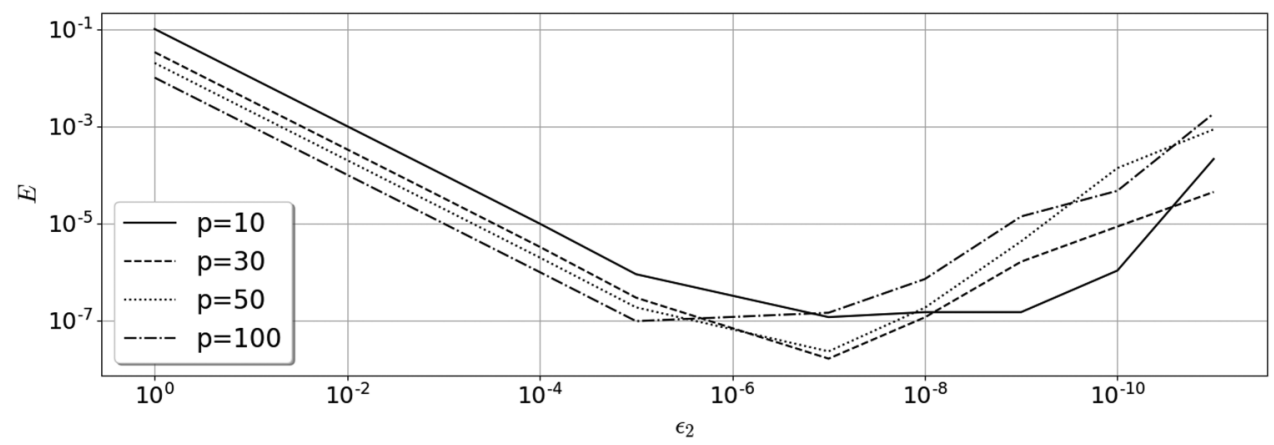

FIG. 3. Comparison between the analytic and numerical sensitivity of the eigenvalue to changes in $\epsilon_{2}$ and the parameter $p$.

A rectangular domain was considered for the generation of an H-grid [Fig. 4(a)]. A mesh convergence analysis for the base flow calculation was performed, showing a mesh independent drag coefficient (up to four digits) for the mesh retained in what follows. Steady symmetrical flow solutions were obtained as a base flow for a Reynolds number equal to 60, and using a symmetric half plane to force steady flow. The Jacobian matrix was extracted from the solution using a discrete approach and fed into the eigenvalue solver. The spectra of this matrix [Fig. 4(b)] shows a pair of complex eigenvalues with positive real part. This unstable pair of eigenvalues is associated with a Hopf bifurcation, characteristic of the von Karman street that can be observed if the flow solution is integrated over time using a transient solver. The sensitivity of the unstable eigenvalue to base flow modifications and steady forcing $\left(\overline{\mathbf{q}}^{+}\right)$are calculated for a Reynolds number of $\operatorname{Re}=60$ using the derived expressions,

$$
\begin{gathered}
\nabla_{\overline{\mathbf{q}}} \sigma=\mathbf{B}^{+}(\widehat{\mathbf{q}}, \mathbf{X}) \widehat{\mathbf{q}}^{+}, \\
\nabla_{\mathbf{q}_{\mathbf{f}}} \sigma=\overline{\mathbf{q}}^{+} .
\end{gathered}
$$

Finally, as the main contribution of this paper, the sensitivity to normal displacement of the surface nodes can be calculated, as stated in Eq. (21). We plot the values of the sensitivity against the azimuth coordinate in Fig. 5(a), together with a geometrical representation of the sensitivity gradients over the cylinder circular shape, nondimensionalized with their maximum absolute value.

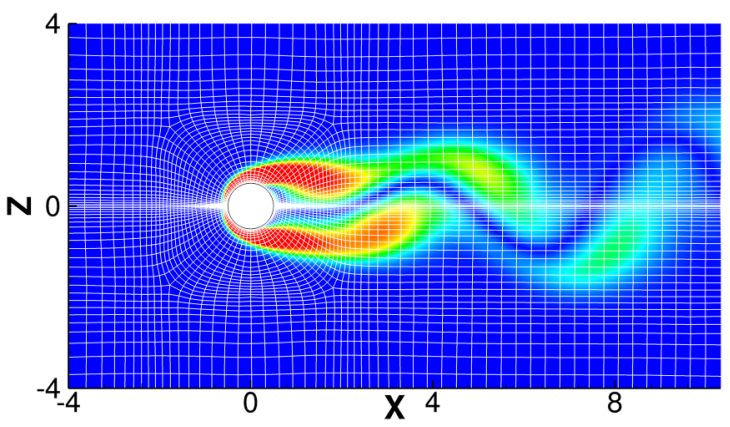

(a)

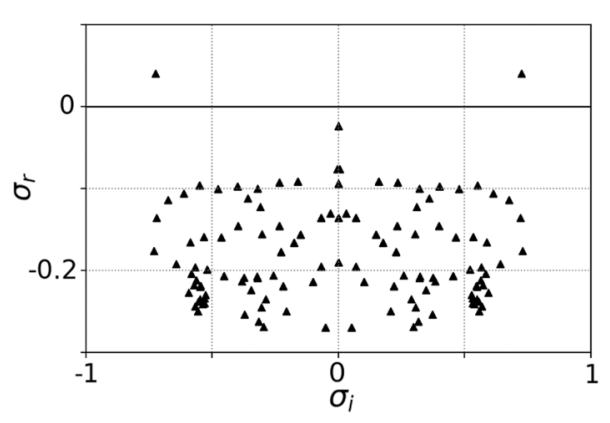

(b)

FIG. 4. (a) Unstructured H-grid used for the simulations, overlapped with vorticity magnitude contours $(\operatorname{Re}=60)$. Only half of the mesh nodes are shown. (b) Eigenvalue distribution for the laminar flow over a cylinder, $\operatorname{Re}=60$. 

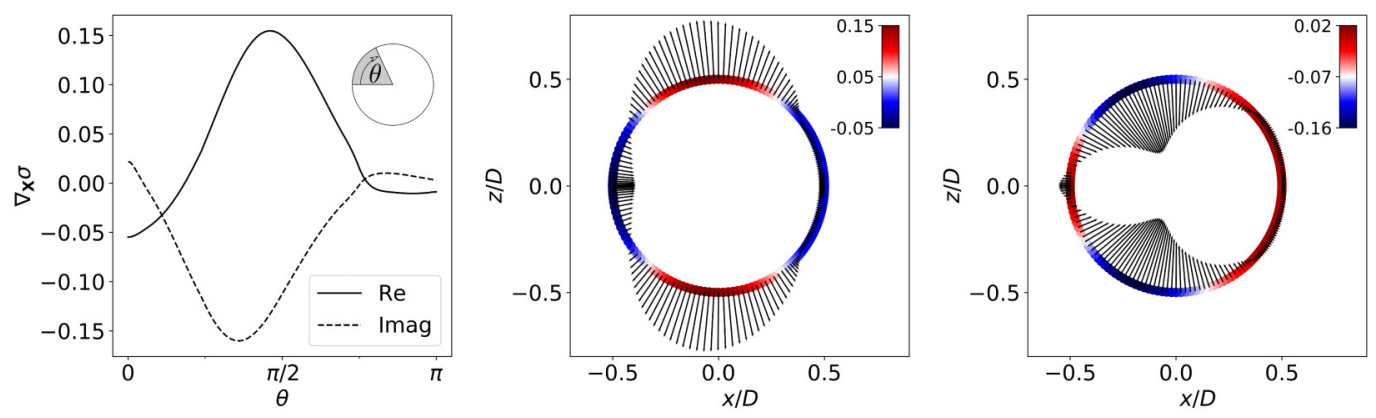

(a) $R e=60$ and $M=0.2$.
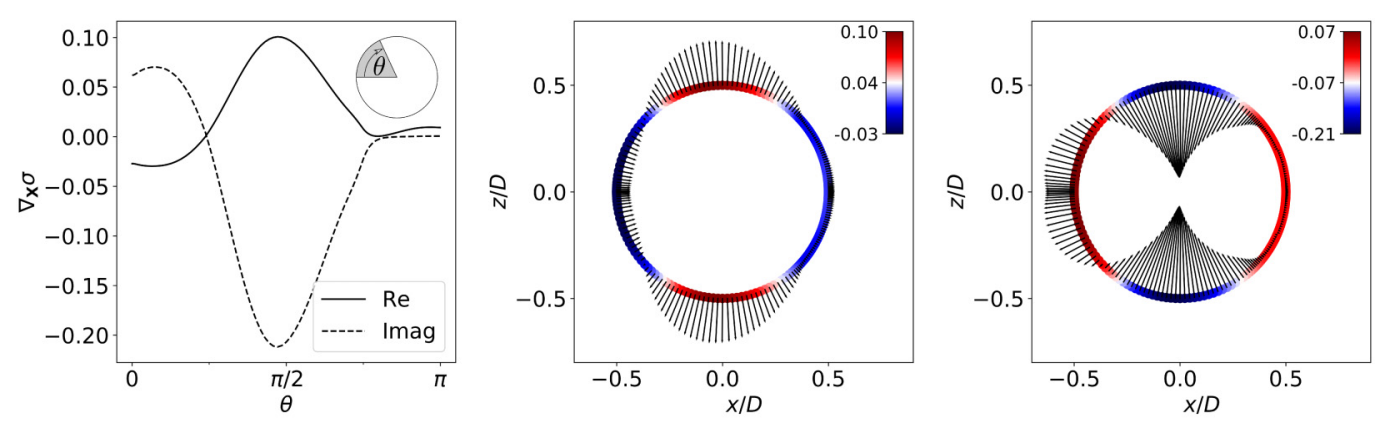

(b) $R e=50$ and $M=0.01$.

FIG. 5. Sensitivity of the eigenvalue to outward normal displacements of the surface nodes, $\nabla_{\mathbf{X}} \sigma$, as a function of the circular cylinder azimuth (left). Node distribution of the sensitivity of the amplification rate (center) and the sensitivity of the pulsation (right) of the eigenvalue.

The real part of the gradient is directly related with the real part of the eigenvalue, meaning that normal displacements of the nodes, following the positive direction of the sensitivity, have a destabilizing impact on the eigenvalue. In addition, the information related to the imaginary part of the gradient is linked to the associated frequency of the eigenvalue (its pulsation, $\sigma_{i}=2 \pi S t$ ), where normal displacements will have an increment or decrement effect on the pulsation, depending on the sign of the gradient. As it can be observed in the figure, the regions located close to $90^{\circ}$ (considering $0^{\circ}$ as the fore stagnation point) are the most sensitive to surface deformations, where a deformation in the outward normal direction implies a further destabilization of the global mode, together with a decrement on the associated frequency. On the contrary, modifications at the aft stagnation area $\left(180^{\circ}\right)$ have a minor effect on the eigenvalue.

For validation purposes, results for near-incompressible flow conditions and a low Reynolds number $(M=0.01$ and $\operatorname{Re}=50)$ are plotted in Fig. 5(b). These results are in good agreement with those recently obtained by Brewster and Juniper [24] for a continuous framework and incompressible flows. Published results agree well with our sensitivities. However, minor differences between the two can be seen in the fore stagnation region, where compressibility effects might not be negligible when using a compressible flow solver. When comparing these results with those of Fig. 5(a), a shift on the sensitivities towards the front of the cylinder is observed for an increase in Mach number. The differences generated by variations on the Reynolds number are smaller, as pictured in Fig. 6(a). Finally, unidirectional deformations are used to validate the shape sensitivity results. Streamwise and cross-stream deformations are performed over the cylinder body, with discrete functions $\Delta \mathbf{x}=\alpha \mathbf{x}$ and $\Delta \mathbf{z}=\alpha \mathbf{z}$, respectively, where $\mathbf{x}$ and $\mathbf{z}$ are the surface mesh node coordinates, and $\alpha$ is a user control parameter to regulate the maximum node deformation. Figure 7 


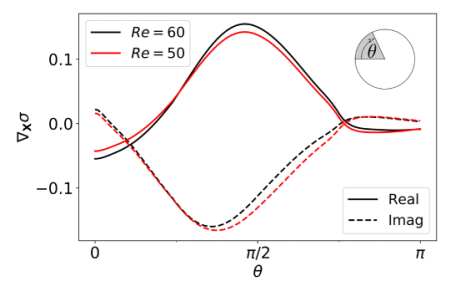

(a)

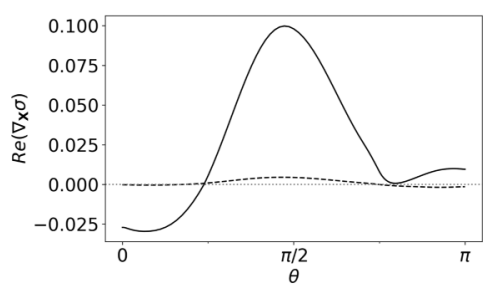

(b)

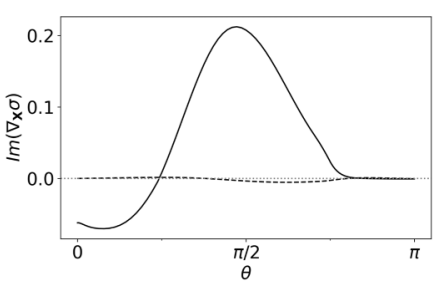

(c)

FIG. 6. (a) Comparison of eigenvalue sensitivity for different Reynolds numbers, $M=0.2$. Eigenvalue (b) growth rate and (c) pulsation sensitivity, split into feedback (dashed line) and base flow (continuous line) contributions $(\operatorname{Re}=50)$.

depicts the linear estimation of the drift of the eigenvalue for $\alpha \in[-0.05,0.05]$, together with the results of the global stability analysis of the recalculated base flow for each deformed body shape. As a last remark, Figs. 6(b) and 6(c) depict the contributions of feedback and base flow contributions of the gradients (as stated in Sec. II B), confirming the observations of Brewster and Juniper [24] that the feedback mechanism contribution plays a minor role compared with the effects generated by modifications on the base flow due to surface deformation, meaning that the control of the instability by surface deformation is provoked by the steady changes generated on the base flow.

The value of the gradient at each surface node shows the contribution to the real or imaginary part of the eigenvalue if that node has a displacement in the normal direction. The real part of the sensitivity gradient [Fig. 5(b)] shows mainly positive values so, from the integration of the area behind the curve of Fig. 5(a), it can be inferred that an increment in the radius of the cylinder will have a destabilizing effect, since this can be associated to an increment of the Reynolds number. Modifications following this gradient would led to a more slender body, either in the direction of the flow or perpendicular to it, and with higher or lower associated frequencies, respectively.

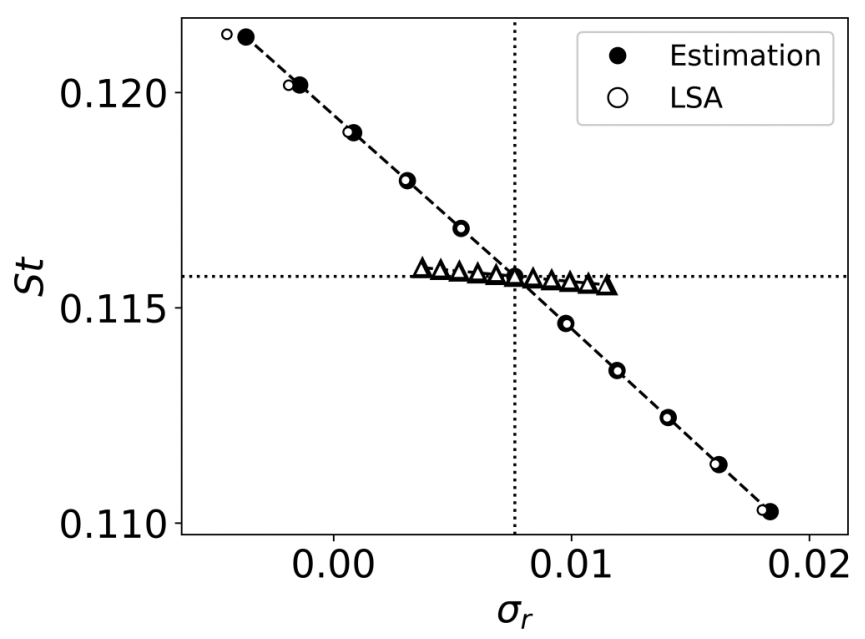

FIG. 7. Drift of the eigenvalue as the cylinder shape is deformed. Linear estimation and stability analysis results of the deformed body shape are compared: $-\circ$ : cross-stream (vertical) deformations; $-\triangle$ : streamwise (horizontal) deformations. The eigenvalue corresponding to the unperturbed body shape is signalized with dotted lines. 


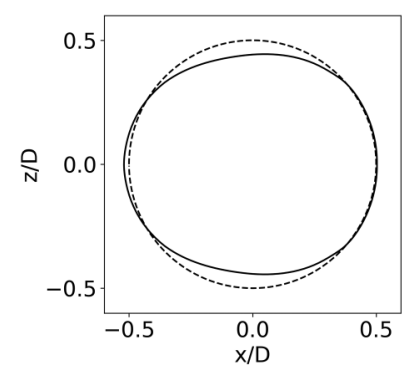

(a)

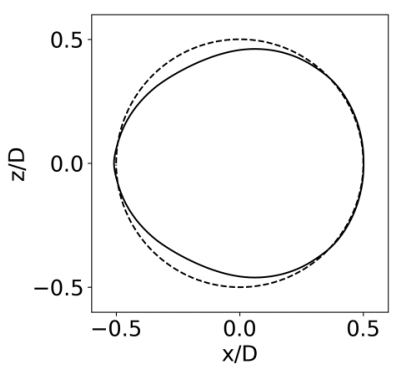

(b)

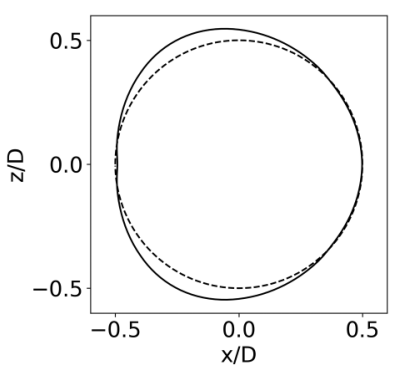

(c)

FIG. 8. Deformed body shapes for (a) more stable configuration, (b) higher associated frequency, and (c) lower associated frequency.

\section{B. Control of the instability}

The control of the global instability related to vortex shedding is typically of major interest, since steady flows decrease fatigue life on structures and minimize noise emissions. To prove the capability of the sensitivity gradients developed in this work, we aim at modifying the growth rate and pulsation of the least stable eigenvalue and, with it, the onset and frequency of the cylinder wake breakdown, by means of local modifications of the circular shape. Note that the objective of this analysis is not to perform an optimization process, but to evaluate the potential of the calculated gradients on the prediction of the directions for shape deformation towards controlling the global instability. We prove their capabilities by evaluating three characteristic deformed configurations, at $\operatorname{Re}=60$ and $M=0.2$. To do so, the cylinder surface mesh is deformed in the direction predicted by the gradients imposing a maximum surface node displacement of $6 \%$ of the cylinder diameter, without setting any restriction to the body volume. The internal radial basis functions (RBFs) mesh deformation module of TAU is used, resulting in a smooth adaptation of the cells far from the body to keep the mesh integrity. The three evaluated configurations are shown in Fig. 8, and are referred to here as shapes (a)-(c).

Shape (a), obtained using the real part of the gradients, has a marginally stable behavior, with the amplification rate of the eigenvalue damped to a negative value. This deformation also results in an increment of the pulsation, which has the opposite effect than the amplification rate, as suggested by Fig. 5(a). The use of the imaginary part of the gradients leads to shapes (b) and (c), allowing for a more subtle control of the associated frequency without stabilizing the global mode. Reduced or larger frontal shape areas are accompanied by an augmented or decreased imaginary part, respectively. Numerical results for the three evaluated modified shapes are gathered in Table I.

The analysis of the recomputed base flows reports significant changes in the flow field after the shape deformation. Configurations (a) and (b) reduce the length of the wake recirculation region, reducing the frontal area and displacing the separation points downstream, and producing a quicker pressure recovery. Moreover, the pressure drop behind the body is weaker in configurations (a) and (b), being this effect is more noticeable in configuration (a). There is an increment in the base

TABLE I. Summary of amplification rate and pulsation values the deformed cylinder body shapes at $\operatorname{Re}=$ $60, M=0.2$.

\begin{tabular}{lcccr}
\hline \hline Geometry & Gradient used & \multicolumn{1}{c}{$\sigma_{r}$} & $\sigma_{i}$ & $\mathrm{St}$ \\
\hline Reference & & 0.041 & 0.726 & 0.116 \\
Lower amp. rate (a) & $\operatorname{Re}\left(\nabla_{\mathbf{X}} \sigma\right)$ & -0.005 & 0.806 & 0.129 \\
Higher frequency (b) & $-\operatorname{Im}\left(\nabla_{\mathbf{X}} \sigma\right)$ & 0.008 & 0.787 & 0.125 \\
Lower frequency (c) & $\operatorname{Im}\left(\nabla_{\mathbf{X}} \sigma\right)$ & 0.048 & 0.647 & 0.103 \\
\hline \hline
\end{tabular}



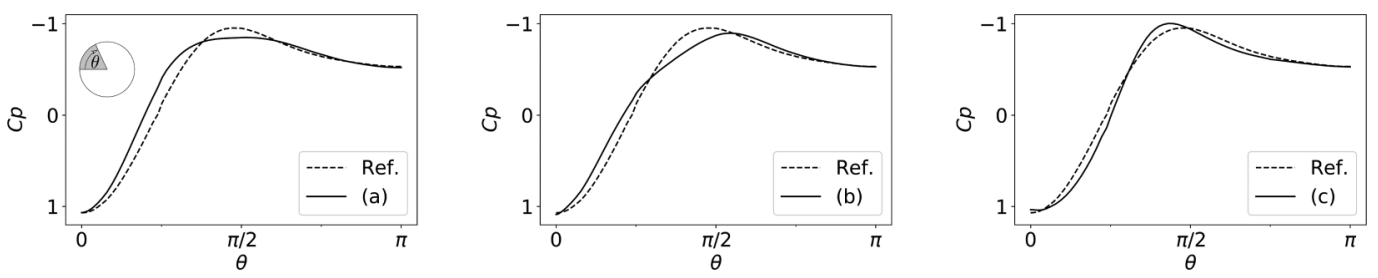

FIG. 9. Pressure coefficient distribution over the cylinder surface for the three optimized configurations. (a) Lower eigenvalue amplification rate, (b) higher frequency, and (c) lower frequency.

pressure caused by the deformation, similar to that expected if a base bleed was applied. This conclusion was also reported by Brewster and Juniper [24] on their analysis of the incompressible cylinder flow. Configuration (c) produces an opposite effect, with a larger recirculation region generated by the increment in the frontal area and its separation points moving upstream with respect to the reference base flow. Changes in the pressure coefficient distribution over the cylinder (Fig. 9) show lower pressure peaks for configurations (a) and (b), which are located downstream in (b). Shape (a) shows a quicker increment in pressure at the frontal area of the cylinder, which later adapts to a low pressure gradient region across the body up to the separation point. This lower surface pressure gradient delays the flow separation and increases the base pressure. On the contrary, configuration (c) presents an early and more significant pressure peak before reaching the top of the cylinder. The enlargement of the frontal area and the displacement of the separation points lead to an increased separation between the lateral shear layers. This effectively reduces the interaction between the shear layers, producing a lower shedding frequency [43].

\section{SURFACE DEFORMATION SENSITIVITY ON COMPRESSIBLE FLOWS: NACA0012 BUFFET INSTABILITY}

One of the main advantages of the presented numerical approach is its capability to tackle general PDE systems, and particularly compressible and turbulent flows. The retained discrete formulation directly introduces the turbulence closure and compressibility terms in the Jacobian matrices, and therefore no additional modifications are needed on the application of the method. To prove the validity of our formulation in complex flows, we perform a numerical analysis of the buffet instability.

Buffet flow instability is one of the aerodynamic phenomena that limits the flight envelope of transonic and supersonic aircraft, as it can develop into structural vibrations (buffeting). Buffeting is nondestructive, but it reduces the structural fatigue life of the aircraft and the aerodynamic performance [44]. When the flow reaches transonic speed over the surface, the interaction between the shock wave and the boundary layer may cause a periodic oscillation of the shock wave that generates varying lift and torque momentum forces on the wing. Experimental and numerical studies of the buffet phenomenon [26,45] identified the Mach number and the angle of incidence of the incoming flow as the triggering parameters of the instability. A two-dimensional linear analysis of the buffet instability over a NACA0012 linking its onset with a global instability was first carried out by Crouch [46], and later on replicated by Iorio et al. [38], who extended the former analysis to the application of adjoint methods and completed the first three-dimensional global stability analysis over an extruded NACA0012 airfoil. Sartor et al. [47] performed a stability and sensitivity analysis over a OAT15 supercritical airfoil, concluding that the global mode leading the instability was very sensitive to flow control within the recirculation region situated downstream of the shock foot, obtaining the optimal forcing distribution to delay or suppress the buffet phenomenon. More recent contributions on this topic include the three-dimensional analysis of the buffet instability from a spanwise periodic assumption of the perturbations [48-50], the use of dynamic mode 

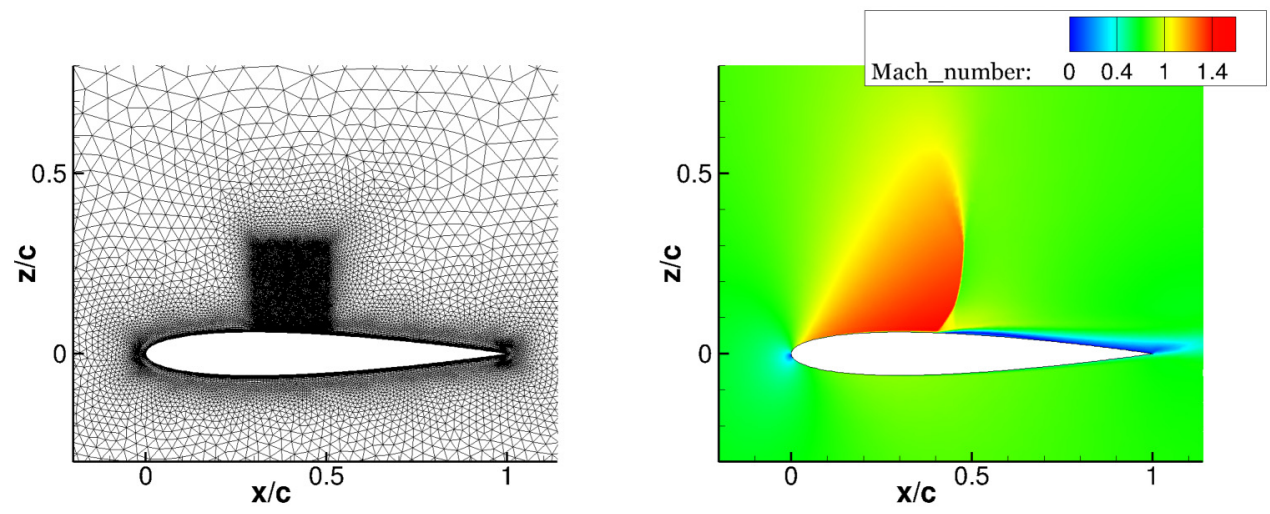

FIG. 10. Illustration of the mesh discretization used on the NACA0012 analysis (left) and Mach number contours of the converged flow solution (right).

decomposition (DMD) on the generation of reduced-order models to predict buffet [51], and a full three-dimensional analysis of a realistic wing-body configuration in transonic flow conditions [52].

In this section, a two-dimensional analysis of a transonic and turbulent flow over a NACA0012 airfoil is replicated, obtaining the corresponding least stable eigenvalues linked to the onset of the buffet instability. The numerical discretization and solver settings are the same used by Iorio et al. [38], using the TAU solver for the computations of the flow solutions and considering the mixing layer compressibility of the Spalart-Allmaras one-equation model [27] for the turbulence closure. The selection of the turbulence model is based on the proven accuracy of the Spalart-Allmaras model on transonic buffet studies over a NACA0012 airfoil $[38,53]$, as this phenomenon has shown to be very sensitive to the turbulence model employed [54]. Figure 10(a) depicts the hybrid mesh used on the analysis, in which the airfoil is embedded inside a circular domain with a radius equal to 100 chords, and a local refinement is used to properly capture the shock wave intensity and position. Details on the mesh generation and the sensitivity of the solution to the spatial discretization can be consulted in Iorio et al. [38]. Figure 10(b) shows the flow solution Mach number contours from the converged steady simulation, at a Reynolds number, Mach number, and angle of incidence of $\operatorname{Re}=10^{7}, M=0.76$, and $\alpha=3.2^{\circ}$, respectively.

As described by Crouch [46], the onset of the buffet instability on a NACA0012 airfoil appears as a Hopf bifurcation, with a pair of complex eigenvalues becoming unstable at an angle of incidence of approximately $\alpha=3.1^{\circ}$. The corresponding unstable direct and adjoint modes from the present simulations are shown in Fig. 11, where the streamwise velocity components of the modes are depicted. Using the adjoint eigenmode, the sensitivity of the leading eigenvalue to steady forcing is calculated as a previous step to the surface deformation gradients, confirming the high sensitivity of the associated global mode to perturbations within the recirculation region and in the upstream boundary layer, depicted in Fig. 13. Our results are in good agreement with those obtained by Sartor et al. [47], where, among other results, it was remarked that an increment in the eddy viscosity of the boundary layer would lead to a more stable eigenvalue. This has special consideration on the stability analysis of transonic buffet, as the turbulence viscosity $\mu_{t}$ must be included on the linearization of the flow solution $[40,52,53]$. To illustrate the sensitivity of the eigenvalue to modifications of the turbulence viscosity, we perturb the flow solution and make use of Eq. (14) to extract the regions where the flow is more sensitive to modifications of this parameter. Figure 12 illustrates a high sensitivity of the eigenvalue if modifications of the eddy viscosity occur at the recirculation region behind the shock wave, in the upstream streamlines of the upper side, or within the boundary layer.

The sensitivity of the eigenvalue to surface node displacements in the normal direction is obtained using Eq. (21), with the results depicted in Fig. 14. The discretized real [Fig. 13(b)] and imaginary 

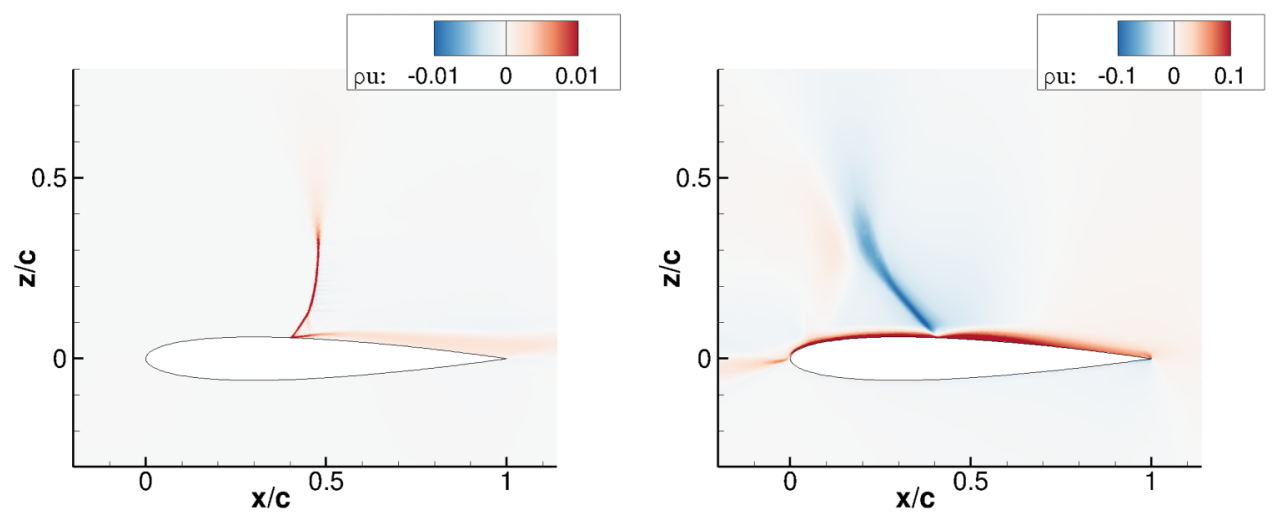

FIG. 11. Direct (left) and adjoint (right) eigenmodes of the NACA0012 buffet global mode. Streamwise momentum perturbations at $\operatorname{Re}=10^{7}, M=0.76$, and $\alpha=3.2^{\circ}$

[Fig. 13(c)] parts of the sensitivity gradients, nondimensionalized with their maximum absolute value, are plotted for each mesh node, showing near zero value in the airfoil, except in the area near the foot of the shock. Figure 13(a) depicts the predicted shape deformation to suppress or delay the onset of buffet together with local Mach number contours. The figure suggests that the smooth bump needs to be placed at the shock wave foot, where the sensitivity reaches its maximum value. The most sensitive regions are linked to the position of the shock wave foot, and therefore the shock control bump (SCB) is optimal only for the specified flow condition. If the design point changes, i.e., to a different free stream Mach number or flow angle of incidence, the calculated deformation is no longer optimal for the suppression of the buffet instability. To address this, Fig. 15 shows the predicted optimal position of the SCB for three different flow conditions, with Mach numbers of $0.76,0.75$, and 0.73 , and flow angles of incidence of $3.2^{\circ}, 3.3^{\circ}$, and $3.8^{\circ}$, respectively, all of them slightly over the buffet envelope [38]. As the Mach number reduces, the angle of incidence needed for the buffet instability to appear increases, which shifts the shock wave position upstream. The

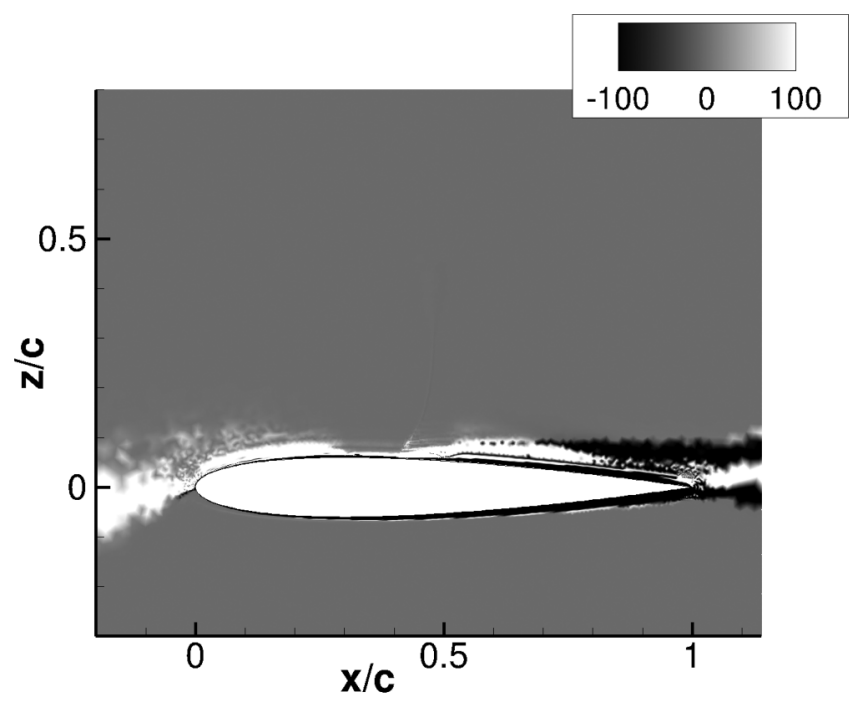

FIG. 12. Contours of the sensitivity of the eigenvalue related to the transonic buffet instability to modifications of the turbulent viscosity, $\mu_{t}$. 

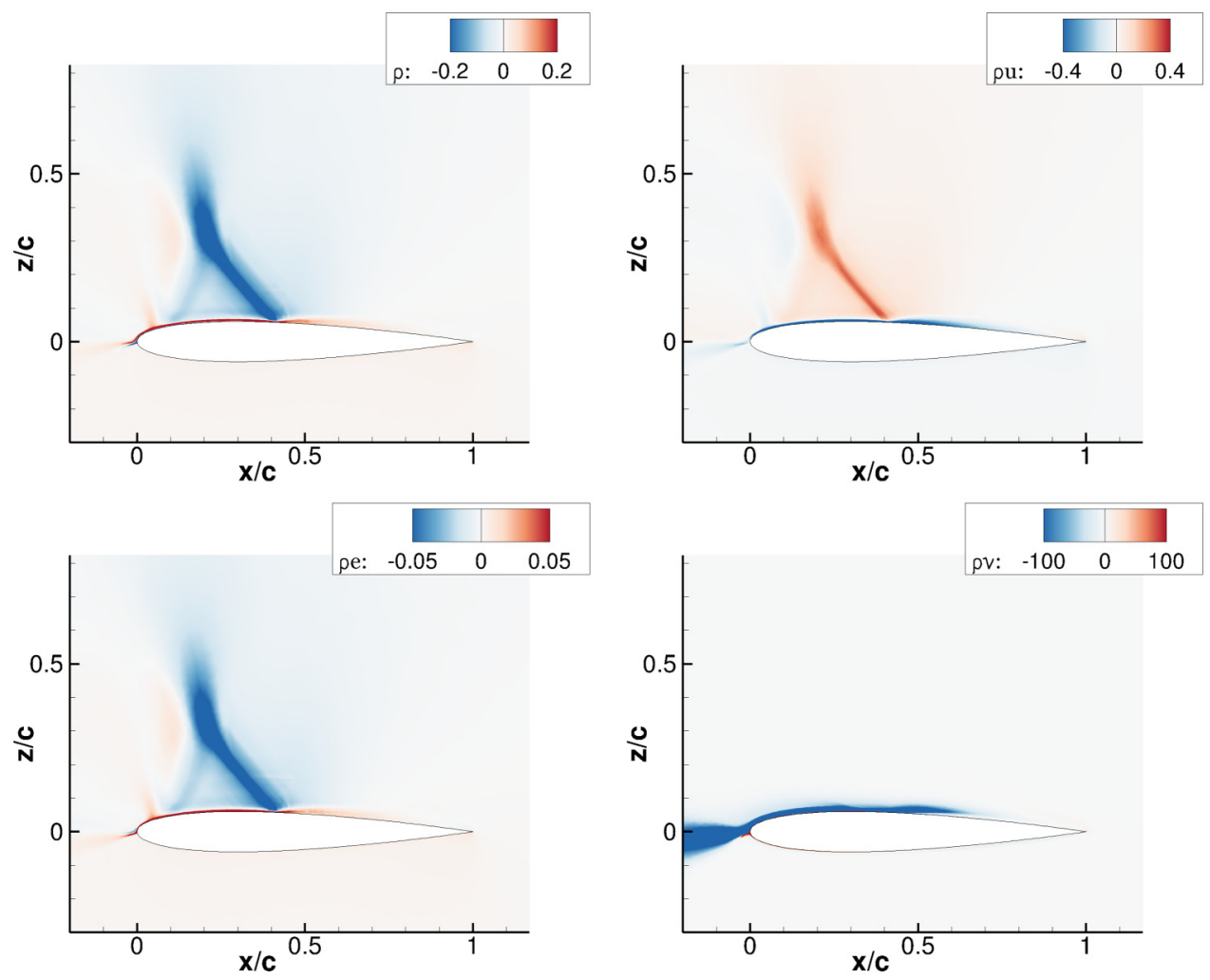

FIG. 13. Sensitivity of the eigenvalue amplification rate to the application of a steady forcing, $\nabla_{f} \sigma_{r}$, at $\operatorname{Re}=10^{7}, M=0.76$, and $\alpha=3.2^{\circ}$.

optimal position for local deformations will hence move upstream as well, following the foot of the shock wave. For this flow configuration, the geometry modifications are valid for the specific operating condition, which is in agreement with Jameson's work [55]. A possible way to circumvent

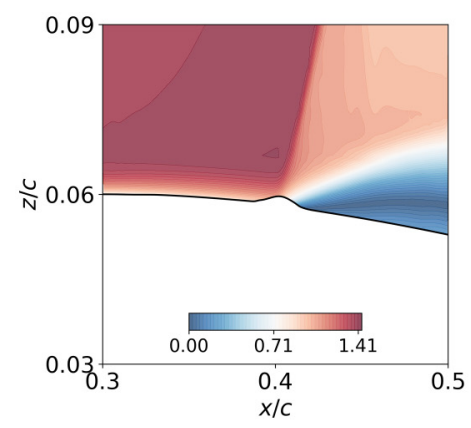

(a)

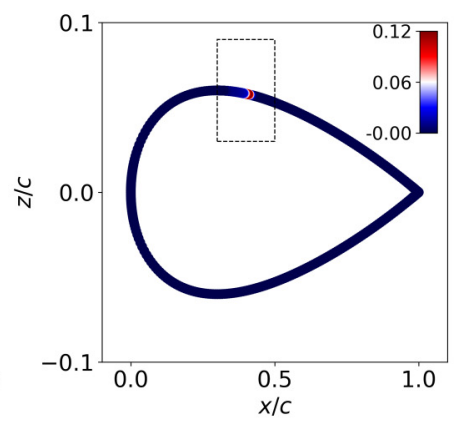

(b)

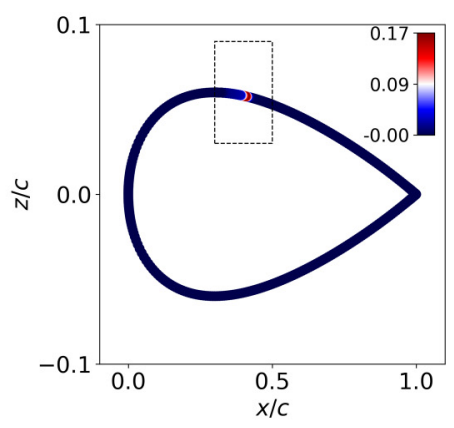

(c)

FIG. 14. Sensitivity of the eigenvalue to outward normal displacements of the surface nodes, $\nabla_{\mathbf{X}} \sigma$. (a) Predicted deformed shape to suppress the instability, with exaggerated height for illustration purposes. Node distribution of the (b) sensitivity of the amplification rate and (c) sensitivity of the pulsation of the eigenvalue. The zoomed area of image (a) is framed by dotted points in (b) and (c). 


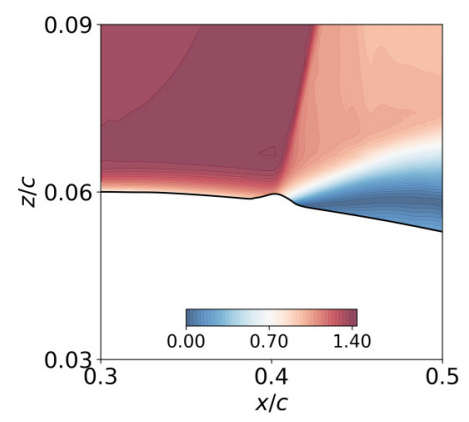

(a)

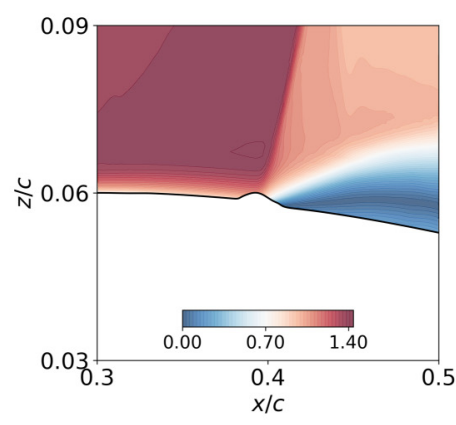

(b)

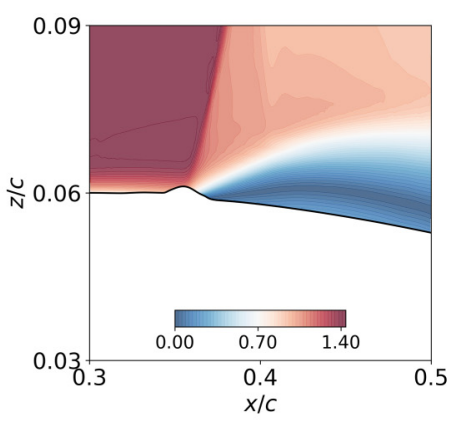

(c)

FIG. 15. Comparison of the optimal position of local deformations for different flow conditions, superimposed into local Mach number contours. (a) $M=0.76, \alpha=3.2^{\circ}$; (b) $M=0.75, \alpha=3.3^{\circ}$; (c) $M=0.73$, $\alpha=3.8^{\circ}$.

this limitation is to design a morphing wing that enables active control on the bumble on the upper surface, but the implementation of such device is out of the scope of this work.

When the airfoil surface is deformed following the gradient direction, the buffet global mode is rapidly damped and the instability suppressed. Figure 16(b) reflects these changes generated on the real and imaginary parts of the eigenvalue when different deformation amplitudes are applied. The global mode is extremely sensitive, with the instability being suppressed with a maximum deformation of only $0.03 \%$ of the airfoil chord. If larger deformations are imposed, reaching maximum values of $0.1 \%$ of the chord length, the onset of the instability can be delayed up to 0.5 of angle of incidence. Interestingly, the predicted deformations to suppress the instability are in line with the well-known shock control bumps (SCBs), developed in recent decades [56]. The placement of a geometrical bump at the foot shock location has shown to suppress or delay the onset of the buffet phenomenon. The curvature of the ramp of the SCB generates a series of oblique compression waves upstream of the original shock foot location, reducing the pressure drop at the shock wave location and hence the shock wave intensity. As predicted by the sensitivity analysis (Fig. 13), this tends to stabilize the global mode. Figure 16(a) shows a plot comparison of the pressure coefficient at the shock foot area for different surface deformations. The shock wave intensity is reduced as the pressure drop is extended in space, widening the shock wave foot across the compression waves. We note that this weakening is accompanied by a drop of the lift and drag coefficients, and results in an increment of the momentum coefficient as well (Table II). Interestingly, the weakening of the

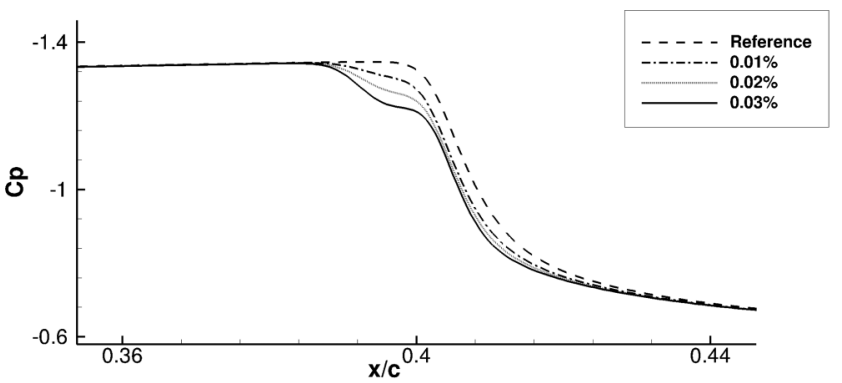

(a)

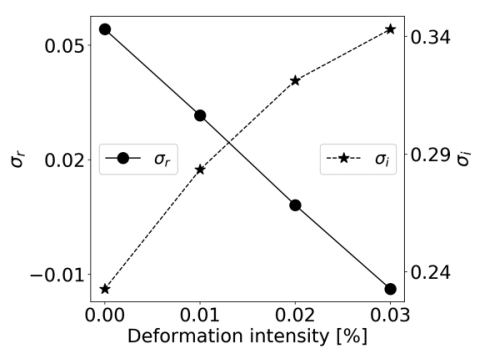

(b)

FIG. 16. (a) Changes in the real and imaginary parts of the eigenvalue with respect to the deformation amplitude. (b) Evolution of the pressure coefficient $\left(C_{p}\right)$ near the shock foot location for different surface deformation amplitude. 
TABLE II. Increments on lift, drag, and momentum coefficients with respect to the surface deformation amplitude.

\begin{tabular}{lccc}
\hline \hline Geometry & $\delta C_{l}(\%)$ & $\delta C_{d}(\%)$ & $\delta C_{m y}(\%)$ \\
\hline Reference (absolute values) & 0.4733 & 0.0357 & 0.0020 \\
$0.01 \%$ deformation & -0.71 & -0.57 & 24.78 \\
$0.02 \%$ deformation & -0.93 & -0.76 & 29.17 \\
$0.03 \%$ deformation & -1.14 & -0.94 & 33.33 \\
\hline \hline
\end{tabular}

shock wave generated by the SCB comes together with an upstream movement of the boundary layer detachment point, increasing the separated flow area. A more stable configuration generated by a weaker shock wave foot can hence deal with larger separated flow area downstream of the shock.

\section{CONCLUSIONS}

We propose a discrete methodology to calculate the gradient sensitivity to arbitrary parameters, which is applicable to any set of PDE including laminar and turbulent RANS flows (e.g., any RANS turbulent model).

Using a Lagrangian methodology and a discrete formulation approach, an eigenvalue sensitivity gradient to geometrical changes has been developed and used to drive a shape optimization process. This eigenvalue-based gradient can be used to control global instabilities in a wide range of compressible and turbulent flows. The approximation provides general parameter gradients that enable the control of the eigenvalue governing the global instability. The proposed methodology can be particularized to obtain the gradient of each surface mesh node of a given geometry in one single computational step, and provides information on the growth rate and frequency enabling the control of each parameter separately. This information is exploited to perform local surface deformations to delay or enhance the onset of instabilities or modify their associated frequency.

First, the methodology has been validated with the well-known global instability of the cylinder wake. The analyses and guided deformations have enabled the suppression of wake oscillators, stabilizing the eigenvalue after maximum mesh node displacements of $6 \%$ of the body diameter at $\mathrm{Re}=60$. In addition, the frequency could be controlled for situations where the flow oscillation does not need to be suppressed, but only modified. The predicted body shapes result in more slender or wider surface shapes, which may alter the base pressure and modify the interaction between the side cylinder's shear layers. Second, the sensitivity gradients have been evaluated for a turbulent compressible flow scenario, enabling the control of the global stability that dominates the buffet phenomenon on a NACA0012 airfoil. The analysis reveals that the instability is very sensitive to surface modifications at the shock wave foot area, needing only deformations of less than $0.04 \%$ of the chord length to suppress it, at $\operatorname{Re}=10^{7}, M=0.76$. The shape modifications predicted by the algorithm are in line with the physical results obtained by the implementation of shock control bumps (obtained by other methods) on the suction side of the wings, which weaken the shock wave at its foot resulting in smoother pressure drops across the shock. The local deformations are therefore only valid for the analyzed operating condition, as the suggested position of the bump is linked to the location of the shock wave foot.

The proposed methodology establishes the basis of an eigenvalue-based surface optimization for compressible and turbulent flows at an affordable cost. Furthermore, the analysis shows the potential of the algorithm to be used in more complex flow conditions and realistic scenarios. 


\section{ACKNOWLEDGMENT}

This research has been funded by the Ministry of Innovation of Spain under the project Simulaciones de alta precisión y modelización para diseño óptimo aeronáutico (SIMOPAIR) (Project No. REF: RTI2018-097075-B-I00).

\section{APPENDIX: DERIVATION OF THE SENSITIVITY FIELDS}

\section{Sensitivity to base flow modifications}

We define the Lagrangian functional as

$$
\mathcal{L}\left(\overline{\mathbf{q}}, \sigma, \widehat{\mathbf{q}}, \lambda_{1}\right)=\sigma+\left\langle\lambda_{1}, \mathbf{A}(\overline{\mathbf{q}}) \widehat{\mathbf{q}}-\sigma \widehat{\mathbf{q}}\right\rangle,
$$

where it will be later confirmed that the Lagrange multiplier $\lambda_{1}$ represents the adjoint eigenmode, $\widehat{\mathbf{q}}^{+}$.

The calculation of the gradient of the Lagrangian gives rise to four different terms,

$$
\nabla \mathcal{L}=\left\langle\frac{\partial \mathcal{L}}{\partial \overline{\mathbf{q}}}, \delta \overline{\mathbf{q}}\right\rangle+\left\langle\frac{\partial \mathcal{L}}{\partial \sigma}, \delta \sigma\right\rangle+\left\langle\frac{\partial \mathcal{L}}{\partial \widehat{\mathbf{q}}}, \delta \widehat{\mathbf{q}}\right\rangle+\left\langle\frac{\partial \mathcal{L}}{\partial \lambda_{1}}, \delta \lambda_{1}\right\rangle
$$

from which we would only want to retain the first term, as we are interested in the influence of the base flow on the eigenvalue.

Cancelling the gradient of the Lagrangian with respect to the Lagrange multiplier $\lambda_{1}$, we recover the standard eigenvalue problem,

$$
\begin{gathered}
\left\langle\frac{\partial \mathcal{L}}{\partial \lambda_{1}}, \delta \lambda_{1}\right\rangle=\left\langle\delta \lambda_{1}, \mathbf{A}(\overline{\mathbf{q}}) \widehat{\mathbf{q}}-\sigma \widehat{\mathbf{q}}\right\rangle=0, \\
\mathbf{A}(\overline{\mathbf{q}}) \widehat{\mathbf{q}}-\sigma \widehat{\mathbf{q}}=0 .
\end{gathered}
$$

The derivation of the Lagrangian with respect to the eigenmode, $\widehat{\mathbf{q}}$, provides the adjoint eigenvalue problem:

$$
\begin{aligned}
&\left\langle\frac{\partial \mathcal{L}}{\partial \widehat{\mathbf{q}}}, \delta \widehat{\mathbf{q}}\right\rangle=\left\langle\lambda_{1},(\mathbf{A}(\overline{\mathbf{q}})-\delta \sigma) \delta \widehat{\mathbf{q}}\right\rangle=\left\langle(\mathbf{A}(\overline{\mathbf{q}})-\delta \sigma)^{+} \lambda_{1}, \delta \widehat{\mathbf{q}}\right\rangle=0, \\
& \mathbf{A}^{+}(\overline{\mathbf{q}}) \lambda_{1}=\sigma^{+} \lambda_{1},
\end{aligned}
$$

from which we can confirm that $\lambda_{1}=\widehat{\mathbf{q}}^{+}$.

The derivation of the Lagrangian with respect to the eigenvalue, $\sigma$, yields a normalization for the adjoint perturbation variable,

$$
\begin{gathered}
\left\langle\frac{\partial \mathcal{L}}{\partial \sigma}, \delta \sigma\right\rangle=\delta \sigma+\left\langle\lambda_{1},-\delta \sigma \widehat{\mathbf{q}}\right\rangle=\delta \sigma-\delta \sigma\left\langle\lambda_{1}, \widehat{\mathbf{q}}\right\rangle=0, \\
\left\langle\lambda_{1}, \widehat{\mathbf{q}}\right\rangle=\left\langle\widehat{\mathbf{q}}^{+}, \widehat{\mathbf{q}}\right\rangle=1 .
\end{gathered}
$$

Finally, the gradient of the Lagrangian with respect to the base flow leads to

$$
\left\langle\frac{\partial \mathcal{L}}{\partial \overline{\mathbf{q}}}, \delta \overline{\mathbf{q}}\right\rangle=\left\langle\lambda_{1}, \frac{\partial \mathbf{A}(\overline{\mathbf{q}}) \widehat{\mathbf{q}}}{\partial \overline{\mathbf{q}}} \delta \overline{\mathbf{q}}\right\rangle=\left\langle\left(\frac{\partial \mathbf{A}(\overline{\mathbf{q}}) \widehat{\mathbf{q}}}{\partial \overline{\mathbf{q}}}\right)^{+} \lambda_{1}, \delta \overline{\mathbf{q}}\right\rangle .
$$

Assuming that the gradient of the Lagrangian and the gradient of $\sigma$ with respect to modifications in the base flow are equal, then

$$
\Delta \sigma=\left\langle\nabla_{\overline{\mathbf{q}}} \sigma, \delta \overline{\mathbf{q}}\right\rangle=\left\langle\frac{\partial \mathcal{L}}{\partial \overline{\mathbf{q}}}, \delta \overline{\mathbf{q}}\right\rangle
$$

Therefore, taking $\lambda_{1}=\widehat{\mathbf{q}}^{+}, \mathbf{B}(\widehat{\mathbf{q}})=\partial(\mathbf{A}(\overline{\mathbf{q}}) \widehat{\mathbf{q}}) / \partial \overline{\mathbf{q}}$ (from now on referred as the sensitivity matrix), expanding the inner products, and applying the adjoint operator previously defined, 
Eq. (A9) becomes

$$
\nabla_{\overline{\mathbf{q}}} \sigma=\mathbf{M}^{-1} \mathbf{B}^{H}(\widehat{\mathbf{q}}) \mathbf{M} \widehat{\mathbf{q}}^{+}
$$

\section{Sensitivity to external forcing}

This time we consider the application of an external force to the system, in the form

$$
\mathbf{M}^{-1} \mathbf{R}(\overline{\mathbf{q}})=\mathbf{q}_{\mathbf{f}} .
$$

This imposes an additional constraint in the Lagrangian form,

$$
\mathcal{L}\left(\overline{\mathbf{q}}, \sigma, \widehat{\mathbf{q}}, \mathbf{q}_{\mathbf{f}}, \lambda_{1}, \lambda_{2}\right)=\sigma+\left\langle\lambda_{1}, \mathbf{A}(\overline{\mathbf{q}}) \widehat{\mathbf{q}}-\sigma \widehat{\mathbf{q}}\right\rangle+\left\langle\lambda_{2}, \mathbf{M}^{-1} \mathbf{R}(\overline{\mathbf{q}})-\mathbf{q}_{\mathbf{f}}\right\rangle,
$$

where it will be later confirmed that the chosen multipliers, $\lambda_{1}$ and $\lambda_{2}$, relate to the adjoint eigenmode and adjoint base flow, $\widehat{\mathbf{q}}^{+}$and $\overline{\mathbf{q}}^{+}$, respectively.

The gradient of the Lagrangian can then be expressed as

$$
\nabla \mathcal{L}=\left\langle\frac{\partial \mathcal{L}}{\partial \overline{\mathbf{q}}}, \delta \overline{\mathbf{q}}\right\rangle+\left\langle\frac{\partial \mathcal{L}}{\partial \sigma}, \delta \sigma\right\rangle+\left\langle\frac{\partial \mathcal{L}}{\partial \widehat{\mathbf{q}}}, \delta \widehat{\mathbf{q}}\right\rangle+\left\langle\frac{\partial \mathcal{L}}{\partial \mathbf{q}_{\mathbf{f}}}, \delta \mathbf{q}_{\mathbf{f}}\right\rangle+\left\langle\frac{\partial \mathcal{L}}{\partial \lambda_{1}}, \delta \lambda_{1}\right\rangle\left\langle\frac{\partial \mathcal{L}}{\partial \lambda_{1}}, \delta \lambda_{2}\right\rangle .
$$

This time, we would like to retain only the fourth component of the Lagrangian, $\partial \mathcal{L} / \partial \mathbf{q}_{\mathbf{f}}$.

Cancelling the gradient of the Lagrangian with respect to the first multiplier, $\lambda_{1}$, leads again to the original eigenvalue problem, and the cancellation with respect to the second multiplier, $\lambda_{2}$, leads to the Navier-Stokes equations modified with the forcing term.

Playing with the third term of the Lagrangian derivative with respect to the eigenmode, we arrive at the adjoint eigenvalue problem, confirming that $\lambda_{1}=\widehat{\mathbf{q}}^{+}$,

$$
\begin{gathered}
\left\langle\frac{\partial \mathcal{L}}{\partial \widehat{\mathbf{q}}}, \delta \widehat{\mathbf{q}}\right\rangle=\left\langle\lambda_{1},(\mathbf{A}(\overline{\mathbf{q}})-\sigma) \delta \widehat{\mathbf{q}}\right\rangle=\left\langle[\mathbf{A}(\overline{\mathbf{q}})-\sigma]^{+} \lambda_{1}, \delta \widehat{\mathbf{q}}\right\rangle=0, \\
\mathbf{A}^{+}(\overline{\mathbf{q}}) \lambda_{1}=\sigma^{+} \lambda_{1} .
\end{gathered}
$$

The derivative of the Lagrangian with respect to the target eigenvalue leads again to a normalization for the adjoint perturbation variable,

$$
\begin{gathered}
\left\langle\frac{\partial \mathcal{L}}{\partial \sigma}, \delta \sigma\right\rangle=\delta \sigma+\left\langle\lambda_{1},-\delta \sigma \widehat{\mathbf{q}}\right\rangle=\delta \sigma-\delta \sigma\left\langle\lambda_{1}, \widehat{\mathbf{q}}\right\rangle=0, \\
\left\langle\widehat{\mathbf{q}}^{+}, \widehat{\mathbf{q}}\right\rangle=1 .
\end{gathered}
$$

This time, the derivative with respect the base flow needs to be canceled as well,

$$
\begin{aligned}
\left\langle\frac{\partial \mathcal{L}}{\partial \overline{\mathbf{q}}}, \delta \overline{\mathbf{q}}\right\rangle & =\left\langle\lambda_{1}, \frac{\partial \mathbf{A}(\overline{\mathbf{q}}) \widehat{\mathbf{q}}}{\partial \mathbf{q}} \delta \overline{\mathbf{q}}\right\rangle+\left\langle\lambda_{2}, \mathbf{M}^{-1} \frac{\partial \mathbf{R}(\overline{\mathbf{q}})}{\partial \mathbf{q}} \delta \overline{\mathbf{q}}\right\rangle \\
& =\left\langle\lambda_{1}, \mathbf{B}(\widehat{\mathbf{q}}) \delta \overline{\mathbf{q}}\right\rangle+\left\langle\lambda_{2}, \mathbf{A}(\overline{\mathbf{q}}) \delta \overline{\mathbf{q}}\right\rangle \\
& =\left\langle\mathbf{B}^{+}(\widehat{\mathbf{q}}) \lambda_{1}, \delta \overline{\mathbf{q}}\right\rangle+\left\langle\mathbf{A}(\overline{\mathbf{q}})^{+} \lambda_{2}, \delta \overline{\mathbf{q}}\right\rangle \\
& =\left\langle\mathbf{B}^{+}(\widehat{\mathbf{q}}) \lambda_{1}+\mathbf{A}(\overline{\mathbf{q}})^{+} \lambda_{2}, \delta \overline{\mathbf{q}}\right\rangle=0 .
\end{aligned}
$$

Replacing $\lambda_{1}$ with $\widehat{\mathbf{q}}^{+}$and inserting Eq. (A11) into the expression above, we retrieve a linear system to obtain $\lambda_{2}$,

$$
\mathbf{A}(\overline{\mathbf{q}})^{+} \lambda_{2}=-\nabla_{\overline{\mathbf{q}}} \sigma
$$

as the product of $\mathbf{B}^{+}(\widehat{\mathbf{q}})$ and $\widehat{\mathbf{q}}^{+}\left(\nabla_{\overline{\mathbf{q}}} \sigma\right)$ is a vector, and the adjoint matrix of the Jacobian of the system has already been defined. This resembles the linear system from the standard adjoint 
approach when the rule of chain is considered,

$$
\left[\frac{\partial \mathbf{R}(\mathbf{q})}{\partial \mathbf{q}}\right]_{\mathbf{q}=\overline{\mathbf{q}}}^{H} \overline{\mathbf{q}}^{+}=-\frac{\partial \sigma}{\partial \overline{\mathbf{q}}},
$$

confirming that $\lambda_{2}$ is the adjoint operator of the base flow, $\overline{\mathbf{q}}^{+}$.

The gradient of the Lagrangian with respect to a steady force $\mathbf{q}_{\mathbf{f}}$ is

$$
\left\langle\frac{\partial \mathcal{L}}{\partial \mathbf{q}_{\mathbf{f}}}, \delta \mathbf{q}_{\mathbf{f}}\right\rangle=\left\langle\lambda_{2}, \delta \mathbf{q}_{\mathbf{f}}\right\rangle .
$$

Finally, we assume that the gradient of the Lagrangian and the gradient of $\sigma$ with respect to a steady force are equal,

$$
\Delta \sigma=\left\langle\nabla_{\mathbf{q}_{\mathbf{f}}} \sigma, \delta \overline{\mathbf{q}}\right\rangle=\left\langle\frac{\partial \mathcal{L}}{\partial \mathbf{q}_{\mathbf{f}}}, \delta \mathbf{q}_{\mathbf{f}}\right\rangle
$$

to obtain

$$
\nabla_{\mathbf{q}_{\mathbf{f}}} \sigma=\overline{\mathbf{q}}^{+} .
$$

To summarize, to extract the sensitivity of the eigenvalue to steady forcing, one sets

$$
\begin{gathered}
\lambda_{1}=\widehat{\mathbf{q}}^{+}, \\
\nabla_{\overline{\mathbf{q}}} \sigma=\mathbf{B}^{+}(\widehat{\mathbf{q}}) \widehat{\mathbf{q}}^{+}, \\
\nabla_{\mathbf{q}_{\mathbf{f}}} \sigma=\lambda_{2}=\overline{\mathbf{q}}^{+} .
\end{gathered}
$$

[1] V. Heuveline and F. Strauß, Shape optimization towards stability in constrained hydrodynamic systems, J. Comput. Phys. 228, 938 (2009).

[2] Y. Kiriyama, E. Katamine, and H. Azegami, Shape optimisation problem for stability of Navier-Stokes flow field, Int. J. Comput. Fluid Dynam. 32, 68 (2018).

[3] Y. Wang, E. Ferrer, A. Martínez-Cava, Y. Zheng, and E. Valero, Enhanced stability of flows through contraction channels: Combining shape optimization and linear stability analysis, Phys. Fluids 31, 074109 (2019).

[4] E. Boujo and F. Gallaire, Controlled reattachment in separated flows: A variational approach to recirculation length reduction, J. Fluid Mech. 742, 618 (2014).

[5] S. Camarri, Flow control design inspired by linear stability analysis, Acta Mech. 226, 979 (2015).

[6] K. Taira, M. S. Hemati, S. L. Brunton, Y. Sun, K. Duraisamy, S. Bagheri, S. T. M. Dawson, and C.-A. Yeh, Modal analysis of fluid flows: Applications and outlook, AIAA J. 58, 998 (2019).

[7] P. J. Schmid and L. Brandt, Analysis of fluid systems: Stability, receptivity, sensitivity, Appl. Mech. Rev. 66, 024803 (2014).

[8] F. Giannetti and P. Luchini, Structural sensitivity of the first instability of the cylinder wake, J. Fluid Mech. 581, 167 (2007).

[9] P. J. Strykowski and K. R. Sreenivasan, On the formation and suppression of vortex 'shedding' at low Reynolds numbers, J. Fluid Mech. 218, 71 (1990).

[10] O. Marquet, D. Sipp, and L. Jacquin, Sensitivity analysis and passive control of cylinder flow, J. Fluid Mech. 615, 221 (2008).

[11] C. Mettot, F. Renac, and D. Sipp, Computation of eigenvalue sensitivity to base flow modifications in a discrete framework: Application to open-loop control, J. Comput. Phys. 269, 234 (2014).

[12] O. M. F. Browne, G. Rubio, E. Ferrer, and E. Valero, Sensitivity analysis to unsteady perturbations of complex flows: A discrete approach, Int. J. Numer. Methods Fluids 76, 1088 (2014). 
[13] P. Meliga, D. Sipp, and J.-M. Chomaz, Open-loop control of compressible afterbody flows using adjoint methods, Phys. Fluids 22, 054109 (2010).

[14] P. Meliga, J.-M. Chomaz, and D. Sipp, Unsteadiness in the wake of disks and spheres: Instability, receptivity and control using direct and adjoint global stability analyses, J. Fluids Struct. 25, 601 (2009).

[15] E. Ferrer, O. M. Browne, and E. Valero, Sensitivity analysis to control the far-wake unsteadiness behind turbines, Energies 10, 1599 (2017).

[16] I. Lashgari, O. Tammisola, V. Citro, M. P. Juniper, and L. Brandt, The planar X-junction flow: Stability analysis and control, J. Fluid Mech. 753, 1 (2014).

[17] P. Luchini and A. Bottaro, Adjoint equations in stability analysis, Annu. Rev. Fluid Mech. 46, 493 (2014).

[18] D. Sipp, O. Marquet, P. Meliga, and A. Barbagallo, Dynamics and control of global instabilities in openflows: A linearized approach, Appl. Mech. Rev. 63, 030801 (2010).

[19] T. Nakazawa and H. Azegami, Shape optimization of flow field improving hydrodynamic stability, Japan J. Indust. Appl. Math. 33, 167 (2016).

[20] O. Tammisola, Optimal wavy surface to suppress vortex shedding using second-order sensitivity to shape changes, Eur. J. Mech. B Fluids 62, 139 (2017).

[21] O. Tammisola, F. Giannetti, V. Citro, and M. P. Juniper, Second-order perturbation of global modes and implications for spanwise wavy actuation, J. Fluid Mech. 755, 314 (2014).

[22] E. Boujo, A. Fani, and F. Gallaire, Second-order sensitivity of parallel shear flows and optimal spanwiseperiodic flow modifications, J. Fluid Mech. 782, 491 (2015).

[23] E. Boujo, A. Fani, and F. Gallaire, Second-order sensitivity in the cylinder wake: Optimal spanwiseperiodic wall actuation and wall deformation, Phys. Rev. Fluids 4, 053901 (2019).

[24] J. Brewster and M. P. Juniper, Shape sensitivity of eigenvalues in hydrodynamic stability, with physical interpretation for the flow around a cylinder, Eur. J. Mech. B Fluids 80, 80 (2020).

[25] D. Barkley and R. Henderson, Three-dimensional Floquet stability analysis of the wake of a circular cylinder, J. Fluid Mech. 322, 215 (1996).

[26] J. B. McDevitt and A. F. Okuno, Static and dynamic pressure measurements on a naca 0012 airfoil in the Ames high Reynolds number facility, NASA Technical Paper, Technical Report No. 2485 (National Aeronautics and Space Administration (NASA), Ames Research Center, United States, 1985).

[27] P. R. Spalart, Trends in turbulence treatments, in Fluids 2000 Conference and Exhibit (American Institute of Aeronautics and Astronautics, Denver, CO, 2000).

[28] M. P. Juniper, Sensitivity analysis of thermoacoustic instability with adjoint Helmholtz solvers, Phys. Rev. Fluids 3, 110509 (2018).

[29] F. Giannetti, S. Camarri, and V. Citro, Sensitivity analysis and passive control of the secondary instability in the wake of a cylinder, J. Fluid Mech. 864, 45 (2019).

[30] D. Schwamborn, T. Gerhold, and R. Heinrich, The DLR Tau-code: Recent applications in research and industry, in Proceedings of the European Conference on Computational Fluid Dynamics, ECCOMAS CFD 2006 (Delft University of Technology, Netherlands, 2006).

[31] R. P. Dwight, Efficiency improvements on RANS-based analysis and optimization using implicit and adjoint methods on unstructured grids, Ph.D. thesis, University of Manchester, 2006.

[32] P. R. Amestoy, I. S. Duff, J.-Y. L'Excellent, and J. Koster, A fully asynchronous multifrontal solver using distributed dynamic scheduling, SIAM J. Matrix Anal. Appl. 23, 15 (2001).

[33] A. Martinez-Cava, Y. Wang, J. de Vicente, and E. Valero, Pressure bifurcation phenomenon on supersonic blowing trailing edges, AIAA J. 57, 153 (2019).

[34] L. M. González, E. Ferrer, and H. R. Díaz-Ojeda, Onset of three-dimensional flow instabilities in liddriven circular, Phys. Fluids 29, 064102 (2017).

[35] C. Mettot, D. Sipp, and H. Bézard, Quasi-laminar stability and sensitivity analyses for turbulent flows: Prediction of low-frequency unsteadiness and passive control, Phys. Fluids 26, 045112 (2014).

[36] V. Hernandez, J. E. Roman, and V. Vidal, SLEPc: A scalable and flexible toolkit for the solution of eigenvalue problems, ACM Trans. Math. Software 31, 351 (2005).

[37] V. Theofilis, Global linear instability, Annu. Rev. Fluid Mech. 43, 319 (2011).

[38] M. C. Iorio, L. M. González, and E. Ferrer, Direct and adjoint global stability analysis of turbulent transonic flows over a naca0012 profile, Int. J. Numer. Methods Fluids 76, 147 (2014). 
[39] S. Sanvido, J. Garicano-Mena, J. de Vicente, and E. Valero, Domain reduction strategy for the stability analysis of complex aerodynamic flows, Int. J. Numer. Methods Fluids 92, 727 (2020).

[40] R. Thormann and M. Widhalm, Linear-frequency-domain predictions of dynamic-response data for viscous transonic flows, AIAA J. 51, 2540 (2013).

[41] B. R. Noack and H. Eckelmann, A global stability analysis of the steady and periodic cylinder wake, J. Fluid Mech. 270, 297 (1994).

[42] D. Canuto and K. Taira, Two-dimensional compressible viscous flow around a circular cylinder, J. Fluid Mech. 785, 349 (2015).

[43] J. H. Gerrard, The mechanics of the formation of vortices behind bluff bodies, J. Fluid Mech. 25, 401 (1966).

[44] D. Caruana, A. Mignosi, M. Corrège, A. Le Pourhiet, and A. M. Rodde, Buffet and buffeting control in transonic flow, Aerospace Sci. Technol. 9, 605 (2005).

[45] G. Barakos and D. Drikakis, Numerical simulation of transonic buffet flows using various turbulence closures, Int. J. Heat Fluid Flow 21, 620 (2000).

[46] J. D. Crouch, A. Garbaruk, and D. Magidov, Predicting the onset of flow unsteadiness based on global instability, J. Comput. Phys. 224, 924 (2007).

[47] F. Sartor, C. Mettot, and D. Sipp, Stability, receptivity, and sensitivity analyses of buffeting transonic flow over a profile, AIAA J. 53, 1980 (2015).

[48] J. D. Crouch, A. Garbaruk, and M. Strelets, Global instability analysis of unswept- and swept-wing transonic buffet onset, in 2018 Fluid Dynamics Conference (American Institute of Aeronautics and Astronautics, Atlanta, GA, 2018).

[49] F. Plante, J. Dandois, S. Beneddine, D. Sipp, and É. Laurendeau, Numerical simulations and global stability analyses of transonic buffet and subsonic stall, 54th 3AF International Conference on Applied Aerodynamics (AAAF Aero 2019) (Association Aéronautique et Astronautique de France, Paris, France, 2019).

[50] E. Paladini, S. Beneddine, J. Dandois, D. Sipp, and J.-C. Robinet, Transonic buffet instability: From two-dimensional airfoils to three-dimensional swept wings, Phys. Rev. Fluids 4, 103906 (2019).

[51] J. Kou, S. Le Clainche, and W. Zhang, A reduced-order model for compressible flows with buffeting condition using higher order dynamic mode decomposition with a mode selection criterion, Phys. Fluids 30, 016103 (2018).

[52] S. Timme, Global instability of wing shock-buffet onset, J. Fluid Mech. 885, A37 (2020).

[53] J. D. Crouch, A. Garbaruk, D. Magidov, and A. Travin, Origin of transonic buffet on aerofoils, J. Fluid Mech. 628, 357 (2009).

[54] M. Thiery and E. Coustols, Numerical prediction of shock induced oscillations over a 2D airfoil: Influence of turbulence modeling and test section walls, Int. J. Heat Fluid Flow 27, 661 (2006).

[55] A. Jameson, L. Martinelli, and N. A. Pierce, Optimum aerodynamic design using the Navier-Stokes equations, Theor. Comput. Fluid Dynam. 10, 213 (1998).

[56] P. J. Bruce and S. P. Colliss, Review of research into shock control bumps, Shock Waves 25, 451 (2015). 